\title{
Taxonomic reassessment and phylogenetic placement of Cyrtodactylus phuketensis (Reptilia, Gekkonidae) based on morphological and molecular evidence
}

\author{
Korkhwan Termprayoon', Attapol Rujirawan', L. Lee Grismer², \\ Perry L. Wood Jr. ${ }^{3}$, Anchalee Aowphol'
}

I Department of Zoology, Faculty of Science, Kasetsart University, Bangkok, 10900, Thailand 2 Herpetology Laboratory, Department of Biology, La Sierra University, 4500 Riverwalk Parkway, Riverside, California 92515, USA 3 Department of Biological Sciences and Museum of Natural History, Auburn University, Auburn, AL, USA

Corresponding author: Anchalee Aowphol (fsciacl@ku.ac.th)

Academic editor: T. Ziegler | Received 10 March 2021 | Accepted 10 May 2021 | Published 28 May 2021

http://zoobank.org/FBD75339-4CE0-45DA-AF56-8E6A263A4BB4

Citation: Termprayoon K, Rujirawan A, Grismer LL, Wood Jr PL, Aowphol A (2021) Taxonomic reassessment and phylogenetic placement of Cyrtodactylus phuketensis (Reptilia, Gekkonidae) based on morphological and molecular evidence. ZooKeys 1040: 91-121. https://doi.org/10.3897/zookeys.1040.65750

\begin{abstract}
The taxonomy and phylogeny of the Cyrtodactylus pulchellus complex along the Thai-Malay Peninsular region has been the focus of many recent studies and has resulted in the recognition of 17 species. However, the majority of these studies were focused on Peninsular and insular Malaysia where there were specimens and genetic vouchers. The taxonomic status and phylogenetic relationships of the Thai species in this complex remain unresolved, due to the lack of genetic material of some species, especially $C$. phuketensis and $C$. macrotuberculatus from Thai populations. In this study, we investigated the phylogenetic relationship between C. phuketensis and its closely related species $C$. macrotuberculatus, using both morphometric and molecular data. Phylogenetic analyses of mitochondrial NADH dehydrogenase subunit 2 (ND2) gene revealed that $C$. phuketensis is embedded within a $C$. macrotuberculatus clade with $1.45-4.20 \%$ (mean $2.63 \%$ ) uncorrected pairwise sequence divergences. Morphological comparisons showed nearly identical measurements of C. phuketensis and C. macrotuberculatus and overlapping ranges in meristic characters. Based on these data, C. phuketensis is considered to be a variant of $C$. macrotuberculatus, thus rendering $C$. phuketensis a junior synonym of $C$. macrotuberculatus.
\end{abstract}

\section{Keywords}

Cyrtodactylus macrotuberculatus, Malaysia, morphology, phylogeny, taxonomic status, Thailand, ThaiMalay Peninsula

Copyright Korkhwan Termprayoon et al. This is an open access article distributed under the terms of the Creative Commons Attribution License (CC BY 4.0), which permits unrestricted use, distribution, and reproduction in any medium, provided the original author and source are credited. 


\section{Introduction}

Cyrtodactylus is a genus of the bent-toed geckos which is widely distributed across South Asia to Melanesia (Wood et al. 2012; Grismer et al. 2020, 2021a). This genus is the most speciose group of gekkotans, with 306 species currently recognized (Uetz et al. 2020). Due to discoveries of hidden taxa within species complexes over decades, species diversity of Cyrtodactylus has remarkably increased, especially in Southeast Asia (e.g., Grismer et al. 2012, 2018; Riyanto et al. 2017; Pauwels et al. 2018; Murdoch et al. 2019; Quah et al. 2019). In the last decade, the integrative approach of molecular and morphological data has been applied to study species boundaries, evaluate taxonomic status, and the adaptive evolution in habitat preference in Cyrtodactylus (Grismer et al. 2015, 2020, 2021a, b; Nielsen and Oliver 2017).

The Cyrtodactylus pulchellus group (Grismer et al. 2021a) is widely distributed along the Thai-Malay Peninsular region and extends from lowland to over 1,500 meters above sea level (Grismer 2011). It was recognized as a single species until morphological differences from an insular population (Pulau Langkawi, Kedah, Malaysia) were noticed and a new species, C. macrotuberculatus Grismer and Ahmad, 2008 was recognized. Several populations of $C$. pulchellus were considered to be part of a species complex that may reveal hidden diversity and unnamed species (Grismer 2011; Grismer et al. 2012). To date, taxonomic revisions of the $C$. pulchellus group have recovered 17 named species based on molecular and morphological data, including C. astrum Grismer et al., 2012, C. australotitiwangsaensis Grismer et al., 2012, C. bintangrendah Grismer et al., 2012, C. bintangtinggi Grismer et al., 2012, C. dayangbuntingensis Quah et al., 2019, C. evanquahi Wood et al., 2020, C. hidupselamanya Grismer et al., 2016, C. jelawangensis Grismer et al., 2014, C. langkawiensis Grismer et al., 2012, C. lekaguli Grismer et al., 2012, C. lenggongensis Grismer et al., 2016, C. macrotuberculatus, C. pulchellus Gray, 1827, C. phuketensis Sumontha et al., 2012 (only morphological data provided), C. sharkari Grismer et al., 2014, C. timur Grismer et al., 2014, and C. trilatofasciatus Grismer et al., 2012. Among this complex group, C. macrotuberculatus and C. phuketensis showed minor morphological differences based on the original description (Sumontha et al. 2012) but no genetic data were provided for elucidating their phylogenetic placement within the $C$. pulchellus group.

Cyrtodactylus phuketensis was described as a new species from Ban Bangrong, Thalang District, Phuket Province by Sumontha et al. (2012). It was similar to C. macrotuberculatus in having tuberculation on ventral surface of the forelimbs, gular region and ventrolateral folds, and relatively larger ventral scales (compared to other species in C. pulchellus complex). In the original description, C. phuketensis was separated from C. macrotuberculatus by having three dark bands between limb insertions, 19 subdigital lamellae on the $4^{\text {th }}$ toe, the presence of a precloacal groove in both sexes, and eight dark caudal bands on an original tail.

During our field surveys, nine specimens of $C$. phuketensis were collected from the type locality and nearby areas and we found variation in the number of body bands and overlap in the ranges of putatively diagnostic meristic characters when compared 
to $C$. macrotuberculatus. Therefore, this study aims to reassess the taxonomic status of C. macrotuberculatus and C. phuketensis using morphological and genetic data from the mitochondrial NADH dehydrogenase subunit 2 (ND2) gene and flanking tRNAs. The analyses were performed on newly collected specimens from southern Thailand and from the type specimens of both species.

\section{Materials and methods}

\section{Specimen sampling}

During October 2017 and June 2019, field surveys were conducted at five localities in southern Thailand, including the type locality of $C$. phuketensis (Fig. 1; Table 1). Specimens were investigated and captured by hand during the night (1900-2300). Liver or muscle tissues were individually preserved in $95 \%$ ethyl alcohol and stored at $-20{ }^{\circ} \mathrm{C}$ for molecular analysis. Specimens were fixed in 10\% formalin and later transferred to $70 \%$ ethyl alcohol. Preserved specimens were deposited in the herpetological collections of the Zoological Museum, Kasetsart University, Thailand (ZMKU). Additional specimens were also examined from the Princess Maha Chakri Sirindhorn Natural History Museum (PSU), Prince of Songkhla University, Thailand; Thailand Natural History Museum (THNHM), Thailand; La Sierra University Herpetological Collection (LSUHC), La Sierra University, Riverside, California, USA; and the Zoological Reference Collection (ZRC) of Lee Kong Chian Natural History Museum at National University of Singapore, Singapore.

\section{Molecular analyses}

Total genomic DNA was extracted from 95\% ethanol-preserved muscle or liver tissue using a NucleoSpin Tissue Kit (Macherey-Nagel GmbH \& Co. KG, Germany). Mitochondrial NADH dehydrogenase subunit 2 (ND2) gene and flanking tRNAs were amplified via double-stand Polymerase Chain Reaction (PCR) using primers L4437a (tRNAmet: 5' AAGCTTTCGGGCCCATACC 3') and H5934 (COI: 5' AGRGTGCCAATGTCTTTGTGRTT 3') (Macey et al. 1997). PCR amplification occurred with an initial denaturation at $94^{\circ} \mathrm{C}$ for $4 \mathrm{~min}$, followed by 35 cycles of denaturation at $94^{\circ} \mathrm{C}$ for $30 \mathrm{sec}$, annealing at $48-52^{\circ} \mathrm{C}$ for $30 \mathrm{sec}$, extension at $72{ }^{\circ} \mathrm{C}$ for $1 \mathrm{~min}$ $30 \mathrm{sec}$, and final extension at $72{ }^{\circ} \mathrm{C}$ for $7 \mathrm{~min}$. Amplification products were purified using NucleoSpin Gel and PCR Clean-Up kit (Macherey-Nagel GmbH \& Co. KG, Germany) and visualized on 1.0\% agarose gel electrophoresis. Purified PCR products were sequenced in both directions using amplifying primers on an ABI 3730XL DNA Sequencer (Applied Biosystems, CA, USA). Sequences were manually edited and aligned in Geneious R11 (Biomatters, Ltd, Auckland, New Zealand). The ND2 nucleotide sequences were translated to amino acid for the protein-coding region and to ensure the lack of stop codons. All sequences were deposited in GenBank under the accession numbers MW809294 to MW809309 (Table 1). 


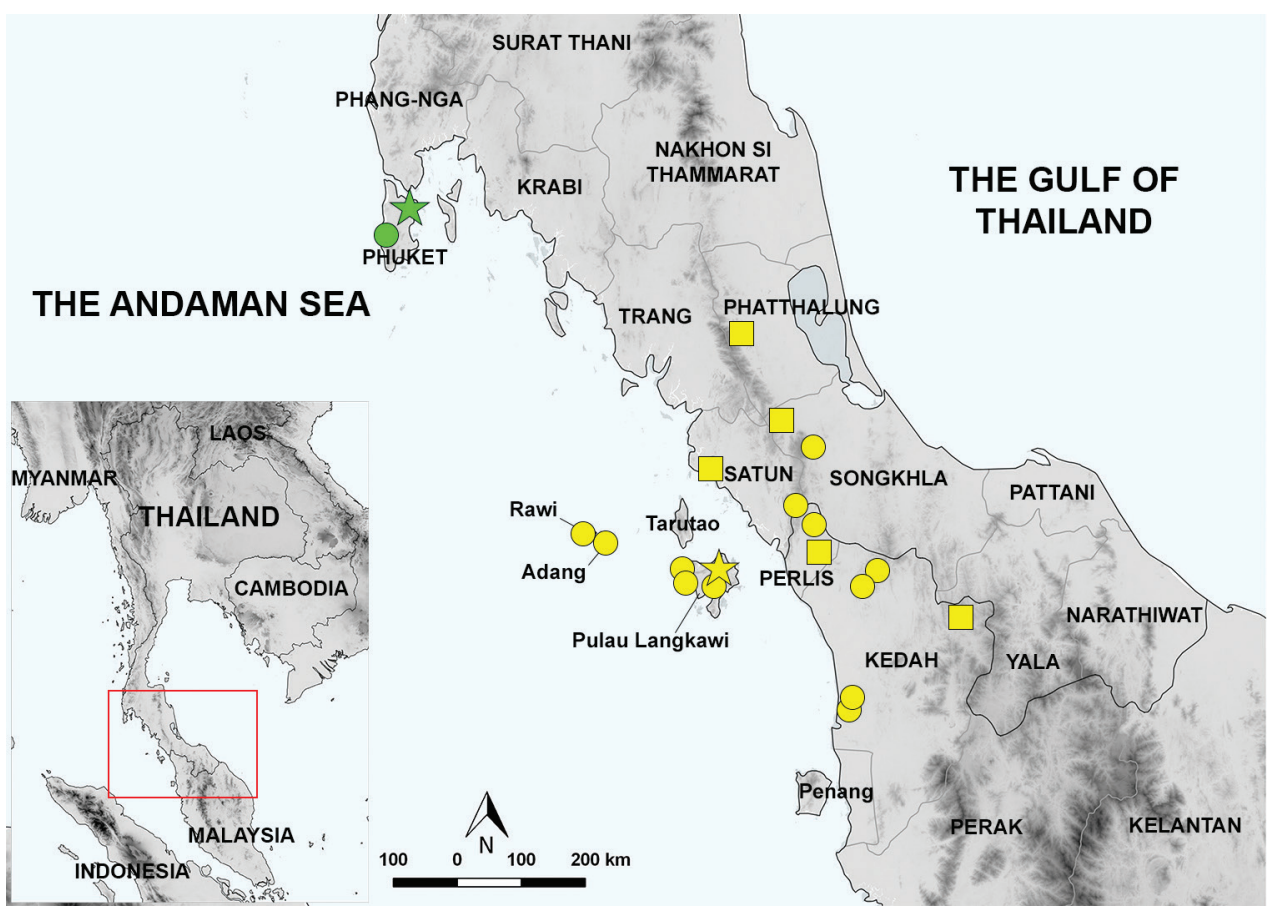

Figure I. Map illustrating the known geographic distribution of Cyrtodactylus macrotuberculatus and C. phuketensis. Yellow star: the type locality of C. macrotuberculatus at Gunung Raya, Pulau Langkawi, Kedah, Malaysia. Green star: the type locality of $C$. phuketensis at Thalang District, Phuket Island, Phuket Province. Yellow circles: C. macrotuberculatus samples used in this study. Green circle: C. phuketensis samples used in this study. Yellow squares: the distribution of $C$. macrotuberculatus taken from Grismer et al. (2012), and Quah et al. (2019). The samples used correspond to those in Table 1.

Phylogenetic relationships were inferred using two model based approaches, Bayesian Inference (BI) and Maximum Likelihood (ML). Outgroup species used to root the tree were Hemidactylus frenatus, Agamura persica, Tropiocolotes steudneri, C. elok, C. intermedius, C. interdigitalis, and Cyrtodactylus sp. based on Wood et al. (2012). The best-fit nucleotide substitution model for each of the three codon partitions and tRNAs was selected under the Bayesian Information Criterion (BIC) in PartitionFinder2 on XSEDE (Lanfear et al. 2016) using CIPRES (Cyberinfrastructure for Phylogenetic Research; Miller et al. 2010). BI analysis was executed in MrBayes 3.2.6 on XSEDE (Ronquist et al. 2012) using CIPRES with the TRN $+\mathrm{I}+\mathrm{G}$ for the $1^{\text {st }}$ and $2^{\text {nd }}$ codon position, and $\mathrm{TIM}+\mathrm{I}+\mathrm{G}$ for the $3^{\text {rd }}$ codon position and tRNAs. Four chains (three hot and one cold) were run for 10,000,000 generations and sampled every 1,000 generations using Markov chain Monte Carlo (MCMC). To build a consensus tree, we discarded the first $25 \%$ of each run as burn-in and assessed stationarity by plotting log-likelihood score in Tracer ver. 1.7.1. (Rambaut et al. 2018). The ML analysis was performed on the web server W-IQ-TREE (Trifinopoulos et al. 2016) with 1,000 bootstrap replicates using ultrafast bootstrap approximation (Minh et al. 2013). Nodes having Bayesian posterior probabilities (BPP) of $\geq 0.95$ and ultrafast bootstrap support (UFB) of $\geq 95$ were considered to be strongly 
supported (Huelsenbeck and Ronquist 2001; Wilcox et al. 2002; Minh et al. 2013). Uncorrected pairwise sequence divergence was calculated to assess within and among species differences using the default settings in MEGA X 10.0.5 (Kumar et al. 2018).

\section{Morphological measurements}

Morphological and meristic characters were modified from the previous studies of Grismer and Ahmad (2008) and Grismer et al. (2012). Measurements were taken with digital calipers to the nearest $0.1 \mathrm{~mm}$ for the following sixteen characters:

SVL snout-vent length, taken from the tip of snout to the vent;

TW tail width, taken at the base of the tail immediately posterior to the postcloacal swelling;

TL tail length, taken from vent to the tip of the tail, original or regenerated;

FL forearm length, taken from the posterior margin of the elbow while flexed $90^{\circ}$ to the inflection of the flexed wrist;

TBL tibia length, taken from the posterior surface of the knee while flexed $90^{\circ}$ to the base of the heel;

AG axilla to groin length, taken from the posterior margin of the forelimb at its insertion point on the body to the anterior margin of the hind limb at its insertion point on the body;

HL head length, the distance from the posterior margin of the retroarticular process of the lower jaw to the tip of the snout;

HW head width, measured at the angle of the jaws;

HD head depth, the maximum height of head from the occiput to the throat;

ED eye diameter, the greatest horizontal diameter of the eye-ball;

EE eye to ear distance, measured from the anterior edge of the ear opening to the posterior edge of the eye-ball;

ES eye to snout distance, measured from anterior most margin of the eye-ball to the tip of snout;

EN eye to nostril distance, measured from the anterior margin of the eye-ball to the posterior margin of the external nares;

IO inter orbital distance, measured between the anterior edges of the orbit;

EL ear length, the greatest vertical distance of the ear opening;

IN internarial distance, measured between the nares across the rostrum.

Additional scale counts and non-meristic characters evaluated were the number of supralabial and infralabial scales counted from the largest scale immediately posterior to the dorsal inflection of the posterior portion of the upper jaw to the rostral and mental scales, respectively; the number of paravertebral tubercles between limb insertions counted in a straight line immediately left of the vertebral column; the number of longitudinal rows of body tubercles counted transversely across the center of the dorsum from one ventrolateral fold to the other; the number of longitudinal rows of ventral scales counted transversely across the center of the abdomen from one ventrolateral fold to 
the other; the presence or absence of tubercles on the ventral surface of the forearm; the presence or absence of tubercles in the gular region, throat, and lateral margins of the abdomen; the number of subdigital lamellae beneath the fourth toe counted from the base of the first phalanx to the claw; the total number of precloacal and femoral pores (i.e., the contiguous rows of femoral and precloacal scales bearing pores combined as a single meristic referred to as the femoroprecloacal pores); the presence or absence of a precloacal depression or groove; the degree of body tuberculation, weak tuberculation referring to dorsal body tubercles that are low and rounded whereas prominent tuberculation refers to tubercles that are raised and keeled; the width of the dark body bands relative to the width of the interspace between the bands; number of dark caudal bands on the original tail; the white caudal bands of adults immaculate or infused with dark pigment; and whether or not the posterior portion of the original tail in hatchlings and juveniles less than $50 \mathrm{~mm}$ SVL was white or whitish and faintly banded or boldly banded.

\section{Morphological analyses}

All statistical analyses were performed using the base statistical software in RStudio v. 1.2.1335 (RStudio Team 2018). To remove potential effects of allometry, mensural characters were scaled to SVL using the following allometric equation: $X_{\text {adj }}=X-\beta(S V L-$ $S L_{\text {mean }}$ ), where $X_{\text {adj }}=$ adjusted value; $X=$ measured value; $\beta=$ unstandardized regression coefficient for each OTU; SVL = measured snout-vent length; $\mathrm{SVL}_{\text {mean }}=$ overall average SVL of each OTU (Thorpe 1975, 1983; Turan 1999; Lleonart et al. 2000). Male and female measurements were analyzed separately to remove potential effects of sexual dimorphism. For morphological analyses, TL (tail length) was excluded due to their different conditions (e.g., original, broken, and regenerated). Importantly, the following type material and topotypic specimens were included in the analysis: $C$. macrotuberculatus (holotype and three paratypes) and C. phuketensis (holotype, paratype and three topotypes). Prior to the morphological analyses, individuals were assigned on the basis of molecular data except $C$. phuketensis based on its distribution into three groups (= species): C. macrotuberculatus, C. phuketensis, and C. pulchellus.

Principal component analysis (PCA) was implemented in the R package FactoMineR (Lê et al. 2008) to discover or reduce dimensionality of the original character variables in order to recover characters bearing the highest degree of variation among groups. Fifteen scaled morphometric and seven meristic characters (scalations) were concatenated and used for the PCA analyses separately by sex. For females, femoroprecloacal pore counts were excluded from the PCA due to their presence in only males.

For univariate analyses, all transformed mensural characters were tested for normality using the Shapiro-Wilk Test. Equality of variances was tested using F-tests. Morphological differences of both males and females between $C$. macrotuberculatus and $C$. phuketensis were examined using a $t$-test (for normally distributed and equal variance data), Welch's $t$-test (for unequal variance data) and Mann-Whitney $U$ test (for non-normally distributed data) at a significant level of 95\%. 


\section{Results}

\section{Phylogenetic relationships}

The aligned matrix contained 1,453 bp of ND2 gene and its flanking tRNAs for 101 samples of the $C$. pulchellus complex including outgroups (Table 1). The standard deviation of split frequencies among the two Bayesian runs was 0.003263, and the Estimated Sample Size (ESS) of all parameters were $\geq 200$. The BI and ML analyses generated similar topologies and strong nodal support for most clades, and only the ML tree is shown (Fig. 2). According to phylogenetic analyses, C. phuketensis is nested within C. macrotuberculatus with strong support (1.00 BPP, $100 \mathrm{UFB}$ ), thus rendering C. macrotuberculatus paraphyletic. Cyrtodactylus macrotuberculatus (including C. phuketensis) was recovered as sister lineage to a clade containing $C$. pulchellus and C. evanquahi. Uncorrected pairwise sequence divergence ( $p$-distance) between $C$. phuketensis and this sister lineage was higher than $8.45 \%$ and within the C. phuketensis and C. macrotuberculatus clade, it ranged from 1.45-4.20\% (mean 2.63\%; Table 2). The $p$-distance within species ranged from $0.00-0.36 \%$ (mean $0.14 \%$ ) for C. phuketensis and $0.00-4.38 \%$ (mean $2.48 \%$ ) for C. macrotuberculatus.

\section{Morphology}

A total of 45 preserved specimens from three species groups (C. macrotuberculatus $=29$, C phuketensis $=10$, and C. pulchellus $=6$ ) were used for principal component analysis (Table 3). The PCA of males showed complete overlap between C. macrotuberculatus and $C$. phuketensis, and they were completely separated from $C$. pulchellus along the first two principal components (Fig. 3A). The first three principal components of males accounted for $53.17 \%$ of the variation. The first principal component (PC1) accounted for $25.78 \%$ of the variation and was most heavily loaded on $\mathrm{HL}_{\text {adj }}$, $\mathrm{ES}_{\text {adj }}, \mathrm{EN}_{\text {adj }}$ and ventral scales; the PC2 accounted for $17.56 \%$ of the variation and was most heavily loaded on $\mathrm{TBL}_{\text {adj }}, \mathrm{IO}_{\text {adj; }}$, supralabial and infralabial scales; and the PC3 accounted for $9.83 \%$ of the variation and was loaded most heavily on longitudinal tubercles (Table 3).

Along the first two PC plots, the PCA of females revealed complete overlap between C. macrotuberculatus and C. phuketensis, which were distinctly separated from C. pulchellus (Fig. 3B). The first three principal components of females accounted for $52.99 \%$ of the variation. The first principal component (PC1) accounted for $23.14 \%$ of the variation and was most heavily loaded on $\mathrm{TW}_{\text {adj }}, \mathrm{AG}_{\mathrm{adj}}, \mathrm{ES}_{\text {adj; }}, \mathrm{EN}_{\text {adj; }}$, ventral scales and number of the $4^{\text {th }}$ toe lamellae; the PC2 accounted for $16.59 \%$ of the variation and was most heavily loaded on $\mathrm{HL}_{\text {adj }}$, $\mathrm{ED}_{\text {adj; }}$, supralabial and infralabial scales; the PC3 accounted for $13.26 \%$ of the variation and was loaded most heavily on $\mathrm{TBL}_{\text {adj }}$ and $\mathrm{EL}_{\text {adj }}$ (Table 3).

Summary univariate statistics of morphological characters of adult males and females are shown in Table 4. In adult males, $C$. macrotuberculatus $(N=18)$ and $C$. phuketensis $(N=6)$ were not significantly different in most morphological characters ( $t$-tests 
Table I. Specimens of Cyrtodactylus used in (A) molecular and/or (B) morphological analyses in this study. $\mathrm{WM}=$ West Malaysia; $\mathrm{TH}=$ Thailand.

\begin{tabular}{|c|c|c|c|c|c|}
\hline Species & Locality & Museum No. & $\begin{array}{c}\text { GenBank } \\
\text { Accession No. }\end{array}$ & $\begin{array}{l}\text { Type of } \\
\text { analysis }\end{array}$ & Reference \\
\hline Hemidactylus frenatus & Unknow & NC 00155 & JX519468 & A & Grismer et al. (2012) \\
\hline Agamura persica & $\begin{array}{c}\text { Pakistan, Baluchistan Province, Makran } \\
\text { District, Gwadar }\end{array}$ & FMNH 247474 & JX440515 & A & Grismer et al. (2012) \\
\hline Tropiocolotes steudneri & Unknow & JB 28 & JX440520 & A & Grismer et al. (2012) \\
\hline Cyrtodactylus elok & WM, Pahang, Fraser's Hill, The Gap & LSUHC 6471 & JQ889180 & A & Grismer et al. (2012) \\
\hline \multirow[t]{3}{*}{ C. intermedius } & $\begin{array}{c}\text { TH, Chantaburi Province, Khao Khitchakut } \\
\text { District }\end{array}$ & LSUHC 9513 & JX519469 & A & Grismer et al. (2012) \\
\hline & $\begin{array}{c}\text { TH, Chantaburi Province, Khao Khitchakut } \\
\text { District }\end{array}$ & LSUHC 9514 & JX519470 & A & Grismer et al. (2012) \\
\hline & Laos, Khammouan Province, Nakai District & FMNH 255454 & JQ889181 & A & Grismer et al. (2012) \\
\hline Cyrtodactylus sp. & TH, Loei, Phu Rua & FMNH 265806 & JX519471 & A & Grismer et al. (2012) \\
\hline \multirow[t]{4}{*}{ C. astrum } & WM, Perlis, Gua Kelam & LSUHC 8806 & JX519481 & A & Grismer et al. (2012) \\
\hline & WM, Perlis, Gua Kelam & LSUHC 8808 & JX519479 & A & Grismer et al. (2012) \\
\hline & WM, Perlis, Kuala Perlis & LSUHC 8815 & JX519482 & A & Grismer et al. (2012) \\
\hline & WM, Perlis, Kuala Perlis & LSUHC 8816 (paratype) & JX519483 & A & Grismer et al. (2012) \\
\hline \multirow[t]{3}{*}{ C. australotitiwangsaensis } & WM, Pahang, Genting Highlands & LSUHC 6637 (holotype) & JX519484 & A & Grismer et al. (2012) \\
\hline & WM, Pahang, Fraser's Hill & LSUHC 8086 & JX519486 & A & Grismer et al. (2012) \\
\hline & WM, Pahang, Fraser's Hill & LSUHC 8087 & JX519485 & A & Grismer et al. (2012) \\
\hline \multirow[t]{4}{*}{ C. bintangrendah } & WM, Kedah, Bukit Palang & LSUHC 9984 & JX519487 & A & Grismer et al. (2012) \\
\hline & WM, Kedah, Bukit Mertajam & LSUHC 10331 (paratype) & MN125076 & A & Quah et al. (2019) \\
\hline & WM, Kedah, Bukit Mertajam & LSUHC 10519 & MN125077 & A & Quah et al. (2019) \\
\hline & WM, Kedah, Bukit Mertajam & LSUHC 10520 (paratype) & MN125078 & A & Quah et al. (2019) \\
\hline \multirow[t]{2}{*}{ C. bintangtinggi } & WM, Perak, Bukit Larut & LSUHC 8862 & JX519493 & A & Grismer et al. (2012) \\
\hline & WM, Perak, Bukit Larut & LSUHC 9006 (paratype) & JX519494 & A & Grismer et al. (2012) \\
\hline \multirow[t]{3}{*}{ C. dayangbuntingensis } & WM, Kedah, Dayang Bunting Island & LSUHC 14353 & MN125090 & A & Quah et al. (2019) \\
\hline & WM, Kedah, Dayang Bunting Island & LSUHC 14354 & MN125091 & A & Quah et al. (2019) \\
\hline & WM, Kedah, Dayang Bunting Island & LSUHC 14355 & MN125092 & A & Quah et al. (2019) \\
\hline \multirow[t]{3}{*}{ C. evanquahi } & WM, Kedah, Gunung Baling & BYU 53435 (holotype) & MN586889 & A & Wood et al. (2020) \\
\hline & WM, Kedah, Gunung Baling & BYU 53436 (paratype) & MN586890 & A & Wood et al. (2020) \\
\hline & WM, Kedah, Gunung Baling & BYU 53437 (paratype) & MN586891 & A & Wood et al. (2020) \\
\hline \multirow[t]{6}{*}{ C. hidupselamanya } & WM, Kelantan, Felda Chiku 7 & LSUHC 12158 (paratype) & KX011412 & A & Grismer et al. (2016) \\
\hline & WM, Kelantan, Felda Chiku 7 & LSUHC 12160 (paratype) & KX011414 & A & Grismer et al. (2016) \\
\hline & WM, Kelantan, Felda Chiku 7 & LSUHC 12161 (paratype) & KX011415 & A & Grismer et al. (2016) \\
\hline & WM, Kelantan, Felda Chiku 7 & LSUHC 12162 (paratype) & KX011416 & A & Grismer et al. (2016) \\
\hline & WM, Kelantan, Felda Chiku 7 & LSUHC 12163 (holotype) & KX011417 & A & Grismer et al. (2016) \\
\hline & WM, Kelantan, Felda Chiku 7 & LSUHC 12173 (paratype) & KX011420 & A & Grismer et al. (2016) \\
\hline \multirow[t]{3}{*}{ C. jelawangensis } & WM, Gunung Stong, Kelantan & LSUHC 11060 (paratype) & KJ659850 & A & Grismer et al. (2014) \\
\hline & WM, Kelantan, Gunung Stong & LSUHC 11061 (paratype) & KJ659851 & A & Grismer et al. (2014) \\
\hline & WM, Gunung Stong, Kelantan & LSUHC 11062 (holotype) & KJ659852 & A & Grismer et al. (2014) \\
\hline \multirow[t]{4}{*}{ C. langkawiensis } & WM, Kedah, Pulau Langkawi, Wat Wanaram & LSUHC 9123 (paratype) & JX519500 & A & Grismer et al. (2012) \\
\hline & WM, Kedah, Pulau Langkawi, Wat Wanaram & LSUHC 9124 (paratype) & JX519499 & A & Grismer et al. (2012) \\
\hline & WM, Kedah, Pulau Langkawi, Wat Wanaram & LSUHC 9125 & JX519496 & A & Grismer et al. (2012) \\
\hline & WM, Kedah, Pulau Langkawi, Wat Wanaram & LSUHC 9435 & JX519495 & A & Grismer et al. (2012) \\
\hline \multirow[t]{4}{*}{ C. lekaguli } & TH, Phang-nga Province, Takua Pa District & ZMKU 00720 & KX011425 & A & Grismer et al. (2016) \\
\hline & TH, Phang-nga Province, Takua Pa District & ZMKU 00721 & KX011426 & A & Grismer et al. (2016) \\
\hline & TH, Phang-nga Province, Takua Pa District & ZMKU 00722 & KX011427 & A & Grismer et al. (2016) \\
\hline & TH, Phang-nga Province, Takua Pa District & ZMKU 00723 & KX011428 & A & Grismer et al. (2016) \\
\hline \multirow[t]{4}{*}{ C. lenggongensis } & WM, Perak, Lenggong Valley & LSUHC 9974 (holotype) & JX519490 & A & Grismer et al. (2012) \\
\hline & WM, Perak, Lenggong Valley & LSUHC 9975 (paratype) & JX519488 & A & Grismer et al. (2012) \\
\hline & WM, Perak, Lenggong Valley & LSUHC 9976 (paratype) & JX519489 & A & Grismer et al. (2012) \\
\hline & WM, Perak, Lenggong Valley & LSUHC 9977 (paratype) & JX519491 & A & Grismer et al. (2012) \\
\hline \multirow[t]{7}{*}{ C. macrotuberculatus } & WM, Kedah, Kuala Nerang & BYU 51869 & MN125085 & A & Quah et al. (2019) \\
\hline & WM, Kedah, Kuala Nerang & BYU 51870 & MN125086 & A & Quah et al. (2019) \\
\hline & WM, Kedah, Gunung Jerai & LSUHC 5939 & JX519513 & A & Grismer et al. (2012) \\
\hline & WM, Kedah, Gunung Jerai & LSUHC 5999 & JX519512 & A & Grismer et al. (2012) \\
\hline & WM, Kedah, Gunung Jerai & LSUHC 6000 & JX519514 & A & Grismer et al. (2012) \\
\hline & $\begin{array}{c}\text { WM, Kedah, Pulau Langkawi, Lubuk } \\
\text { Sembilang }\end{array}$ & LSUHC 6829 & JX519505 & A & Grismer et al. (2012) \\
\hline & $\begin{array}{c}\text { WM, Kedah, Pulau Langkawi, Gunung } \\
\text { Machinchang }\end{array}$ & LSUHC 7560 & JX519503 & A & Grismer et al. (2012) \\
\hline
\end{tabular}




\begin{tabular}{|c|c|c|c|c|c|}
\hline Species & Locality & Museum No. & $\begin{array}{c}\text { GenBank } \\
\text { Accession No. }\end{array}$ & $\begin{array}{l}\text { Type of } \\
\text { analysis }\end{array}$ & Reference \\
\hline \multirow[t]{40}{*}{ C. macrotuberculatus } & WM, Kedah, Pulau Langkawi, Gunung Raya & LSUHC 9428 & JX519506 & A, B & $\begin{array}{l}\text { Grismer et al. } \\
\text { (2012), This study }\end{array}$ \\
\hline & WM, Kedah, Pulau Langkawi, Gunung Raya & LSUHC 9429 & - & B & This study \\
\hline & WM, Kedah, Pulau Langkawi, Gunung Raya & LSUHC 9432 & - & B & This study \\
\hline & $\begin{array}{c}\text { WM, Kedah, Pulau Langkawi, Gunung } \\
\text { Machinchang }\end{array}$ & LSUHC 9448 & JX519507 & A & Grismer et al. (2012) \\
\hline & $\begin{array}{c}\text { WM, Kedah, Pulau Langkawi, Gunung } \\
\text { Machinchang }\end{array}$ & LSUHC 9449 & JX519509 & A & Grismer et al. (2012) \\
\hline & WM, Kedah, Hutan Lipur Sungai Tupah & LSUHC 9671 & JX519510 & A & Grismer et al. (2012) \\
\hline & WM, Kedah, Hutan Lipur Sungai Tupah & LSUHC 9672 & JX519511 & A & Grismer et al. (2012) \\
\hline & WM, Kedah, Hutan Lipur Sungai Tupah & LSUHC 9693 & JX519517 & A & Grismer et al. (2012) \\
\hline & WM, Perlis, Perlis State Park & LSUHC 9980 & JX519515 & A & Grismer et al. (2012) \\
\hline & WM, Perlis, Perlis State Park & LSUHC 9981 & JX519516 & A, B & $\begin{array}{l}\text { Grismer et al. } \\
\text { (2012), This study }\end{array}$ \\
\hline & WM, Perlis, Bukit Chabang & LSUHC 10037 & JX519519 & A & Grismer et al. (2012) \\
\hline & WM, Perlis, Bukit Chabang & LSUHC 10038 & JX519518 & A & Grismer et al. (2012) \\
\hline & WM, Perlis, Perlis State Park & LSUHC 10067 & - & B & This study \\
\hline & WM, Kedah, Bukit Wang & LSUHC 10329 & MN125088 & A & Quah et al. (2019) \\
\hline & WM, Kedah, Bukit Wang & LSUHC 10330 & MN125087 & A & Quah et al. (2019) \\
\hline & WM, Perlis, Perlis State Park & ZRC 2.4869 & - & B & This study \\
\hline & WM, Kedah, Pulau Langkawi, Gunung Raya & ZRC 2.6754 (holotype) & - & B & This study \\
\hline & WM, Kedah, Pulau Langkawi, Gunung Raya & ZRC 2.6755 (paratype) & - & B & This study \\
\hline & WM, Kedah, Pulau Langkawi, Gunung Raya & ZRC 2.6756 (paratype) & - & B & This study \\
\hline & WM, Kedah, Pulau Langkawi, Telaga Tujuh & $\begin{array}{l}\text { ZRC 2.6757/ LSUHC } \\
7173 \text { (paratype) }\end{array}$ & JX519508 & A & Grismer et al. (2012) \\
\hline & $\begin{array}{c}\text { WM, Kedah, Pulau Langkawi, Lubuk } \\
\text { Semilang }\end{array}$ & ZRC 2.6758 (paratype) & - & B & This study \\
\hline & $\begin{array}{c}\text { TH, Satun Province, Mueang Satun District, } \\
\text { Adang Island }\end{array}$ & ZMKU R 00871 & - & B & This study \\
\hline & $\begin{array}{c}\text { TH, Satun Province, Mueang Satun District, } \\
\text { Adang Island }\end{array}$ & ZMKU R 00872 & - & B & This study \\
\hline & $\begin{array}{c}\text { TH, Satun Province, Mueang Satun District, } \\
\text { Adang Island }\end{array}$ & ZMKU R 00873 & - & B & This study \\
\hline & $\begin{array}{c}\text { TH, Satun Province, Mueang Satun District, } \\
\text { Adang Island }\end{array}$ & ZMKU R 00874 & MW809294 & A, B & This study \\
\hline & $\begin{array}{c}\text { TH, Satun Province, Mueang Satun District, } \\
\text { Adang Island }\end{array}$ & ZMKU R 00875 & MW809295 & A, B & This study \\
\hline & $\begin{array}{c}\text { TH, Songkhla Province, Hat Yai District, } \\
\text { Thung Tam Sao }\end{array}$ & ZMKU R 00876 & MW809296 & A, B & This study \\
\hline & $\begin{array}{c}\text { TH, Songkhla Province, Hat Yai District, } \\
\text { Thung Tam Sao }\end{array}$ & ZMKU R 00877 & MW809297 & A, B & This study \\
\hline & $\begin{array}{c}\text { TH, Songkhla Province, Hat Yai District, } \\
\text { Thung Tam Sao }\end{array}$ & ZMKU R 00878 & MW809298 & A, B & This study \\
\hline & $\begin{array}{c}\text { TH, Satun Province, Mueang Satun District, } \\
\text { Adang Island }\end{array}$ & ZMKU R 00879 & - & B & This study \\
\hline & $\begin{array}{c}\text { TH, Satun Province, Mueang Satun District, } \\
\text { Adang Island }\end{array}$ & ZMKU R 00880 & - & B & This study \\
\hline & $\begin{array}{c}\text { TH, Satun Province, Mueang Satun District, } \\
\text { Adang Island }\end{array}$ & ZMKU R 00881 & - & B & This study \\
\hline & $\begin{array}{c}\text { TH, Satun Province, Mueang Satun District, } \\
\text { Adang Island }\end{array}$ & ZMKU R 00882 & - & B & This study \\
\hline & $\begin{array}{c}\text { TH, Satun Province, Mueang Satun District, } \\
\text { Rawi Island }\end{array}$ & ZMKU R 00883 & MW809299 & A, B & This study \\
\hline & $\begin{array}{c}\text { TH, Satun Province, Mueang Satun District, } \\
\text { Rawi Island }\end{array}$ & ZMKU R 00884 & - & B & This study \\
\hline & $\begin{array}{c}\text { TH, Satun Province, Mueang Satun District, } \\
\text { Rawi Island }\end{array}$ & ZMKU R 00885 & - & B & This study \\
\hline & $\begin{array}{c}\text { TH, Satun Province, Mueang Satun District, } \\
\text { Rawi Island }\end{array}$ & ZMKU R 00886 & - & B & This study \\
\hline & $\begin{array}{c}\text { TH, Satun Province, Mueang Satun District, } \\
\text { Rawi Island }\end{array}$ & ZMKU R 00887 & MW809300 & A, B & This study \\
\hline & $\begin{array}{c}\text { TH, Satun Province, Mueang Satun District, } \\
\text { Rawi Island }\end{array}$ & ZMKU R 00888 & - & B & This study \\
\hline & $\begin{array}{l}\text { TH, Satun Province, Mueang Satun District, } \\
\text { Rawi Island }\end{array}$ & ZMKU R 00889 & - & B & This study \\
\hline
\end{tabular}




\begin{tabular}{|c|c|c|c|c|c|}
\hline Species & Locality & Museum No. & $\begin{array}{c}\text { GenBank } \\
\text { Accession No. }\end{array}$ & $\begin{array}{r}\text { Type of } \\
\text { analysis }\end{array}$ & Reference \\
\hline \multirow[t]{11}{*}{$\begin{array}{l}\text { C. macrotuberculatus } \\
\text { (as C. phuketensis) }\end{array}$} & $\begin{array}{c}\text { TH, Phuket Province, Kathu District, Kathu } \\
\text { Waterfall }\end{array}$ & ZMKU R 00890 & MW809301 & $\mathrm{A}, \mathrm{B}$ & This study \\
\hline & $\begin{array}{c}\text { TH, Phuket Province, Kathu District, Kathu } \\
\text { Waterfall }\end{array}$ & ZMKU R 00891 & MW809302 & A, B & This study \\
\hline & $\begin{array}{c}\text { TH, Phuket Province, Kathu District, Kathu } \\
\text { Waterfall }\end{array}$ & ZMKU R 00892 & MW809303 & A, B & This study \\
\hline & $\begin{array}{c}\text { TH, Phuket Province, Kathu District, Kathu } \\
\text { Waterfall }\end{array}$ & ZMKU R 00893 & MW809304 & A, B & This study \\
\hline & $\begin{array}{c}\text { TH, Phuket Province, Thalang District, Thep } \\
\text { Krasatti }\end{array}$ & ZMKU R 00894 & MW809305 & A, B & This study \\
\hline & $\begin{array}{c}\text { TH, Phuket Province, Thalang District, Thep } \\
\text { Krasatti }\end{array}$ & ZMKU R 00895 & MW809306 & A, B & This study \\
\hline & $\begin{array}{c}\text { TH, Phuket Province, Thalang District, Thep } \\
\text { Krasatti }\end{array}$ & ZMKU R 00896 & MW809307 & A, B & This study \\
\hline & $\begin{array}{c}\text { TH, Phuket Province, Kathu District, Kathu } \\
\text { Waterfall }\end{array}$ & ZMKU R 00897 & MW809308 & A, B & This study \\
\hline & $\begin{array}{c}\text { TH, Phuket Province, Kathu District, Kathu } \\
\text { Waterfall }\end{array}$ & ZMKU R 00898 & MW809309 & A & This study \\
\hline & $\begin{array}{c}\text { TH, Phuket Province, Thalang District, Thep } \\
\text { Krasatti }\end{array}$ & PSUZC-RT 2010.58 & - & B & This study \\
\hline & $\begin{array}{c}\text { TH, Phuket Province, Thalang District, Thep } \\
\text { Krasatti }\end{array}$ & THNHM 15378 & - & B & This study \\
\hline \multirow[t]{11}{*}{ C. pulchellus } & $\begin{array}{l}\text { WM, Penang, Pulau Pinang, Empangan Air } \\
\text { Itam }\end{array}$ & LSUHC 6668 & JX519523 & A & Grismer et al. (2012) \\
\hline & WM, Penang, Pulau Pinang, Moongate Trail & LSUHC 6726 & JX519527 & A & Grismer et al. (2012) \\
\hline & WM, Penang, Pulau Pinang, Moongate Trail & LSUHC 6727 & JX519526 & A, B & $\begin{array}{l}\text { Grismer et al. } \\
\text { (2012), This study }\end{array}$ \\
\hline & WM, Penang, Pulau Pinang, Moongate Trail & LSUHC 6728 & JX519525 & A, B & $\begin{array}{l}\text { Grismer et al. } \\
\text { (2012), This study }\end{array}$ \\
\hline & WM, Penang, Pulau Pinang, Moongate Trail & LSUHC 6729 & JX519528 & A, B & $\begin{array}{l}\text { Grismer et al. } \\
\text { (2012), This study }\end{array}$ \\
\hline & WM, Penang, Pulau Pinang, Moongate Trail & LSUHC 6785 & JX519524 & A & Grismer et al. (2012) \\
\hline & $\begin{array}{c}\text { WM, Penang, Pulau Pinang, Air Terjun Titi } \\
\text { Kerawang }\end{array}$ & LSUHC 9667 & JX519520 & A & Grismer et al. (2012) \\
\hline & $\begin{array}{l}\text { WM, Penang, Pulau Pinang, Air Terjun Titi } \\
\text { Kerawang }\end{array}$ & LSUHC 9668 & JX519521 & A & Grismer et al. (2012) \\
\hline & $\begin{array}{c}\text { WM, Penang, Pulau Pinang, Air Terjun Titi } \\
\text { Kerawang }\end{array}$ & LSUHC 10022 & JX519522 & A, B & $\begin{array}{l}\text { Grismer et al. } \\
\text { (2012), This study }\end{array}$ \\
\hline & WM, Penang, Pulau Pinang & ZRC 2.4854 & - & B & This study \\
\hline & WM, Penang, Pulau Pinang & ZRC 2.4857 & - & B & This study \\
\hline C. sharkari & WM, Pahang, Merapoh, Gua Gunting & LSUHC 11022 (holotype) & KJ659853 & A & Grismer et al. (2014) \\
\hline \multirow[t]{4}{*}{ C. timur } & WM, Gunung Tebu, Terengganu & LSUHC 10886 & KJ659854 & A & Grismer et al. (2014) \\
\hline & WM, Gunung Tebu, Terengganu & LSUHC 11183 (paratype) & KJ659855 & A & Grismer et al. (2014) \\
\hline & WM, Gunung Tebu, Terengganu & LSUHC 11184 (paratype) & KJ659856 & A & Grismer et al. (2014) \\
\hline & WM, Gunung Tebu, Terengganu & LSUHC 11185 (paratype) & KJ659857 & A & Grismer et al. (2014) \\
\hline \multirow[t]{3}{*}{ C. trilatofasciatus } & WM, Pahang, Cameron Highlands & LSUHC 10064 & JX519529 & A & Grismer et al. (2012) \\
\hline & WM, Pahang, Cameron Highlands & LSUHC 10065 & JX519530 & A & Grismer et al. (2012) \\
\hline & WM, Pahang, Cameron Highlands & LSUHC 10066 & JX519531 & A & Grismer et al. (2012) \\
\hline
\end{tabular}

Table 2. Percentage uncorrected pairwise sequence divergence ( $p$-distance) for Cyrtodactylus macrotuberculatus, $C$. phuketensis, and closely related species calculated from 1,453 base pairs of the mitochondrial gene ND2 and the flanking tRNAs. Numbers in bold represent the mean and the range of within species $p$-distances.

\begin{tabular}{|c|c|c|c|c|c|c|}
\hline & Species & $N$ & 1 & 2 & 3 & 4 \\
\hline 1 & C. pulchellus & 9 & $1.02(0.14-2.20)$ & & & \\
\hline 2 & C. evanquahi & 3 & $7.42(6.64-8.38)$ & $0.24(0.14-0.36)$ & & \\
\hline 3 & C. macrotuberculatus & 27 & $8.93(7.47-10.48)$ & $8.08(6.64-8.38)$ & $2.48(0.00-4.38)$ & \\
\hline 4 & C. phuketensis & 9 & $9.41(8.71-10.29)$ & $8.79(8.45-8.95)$ & $2.63(1.45-4.20)$ & $0.14(0.00-0.36)$ \\
\hline
\end{tabular}



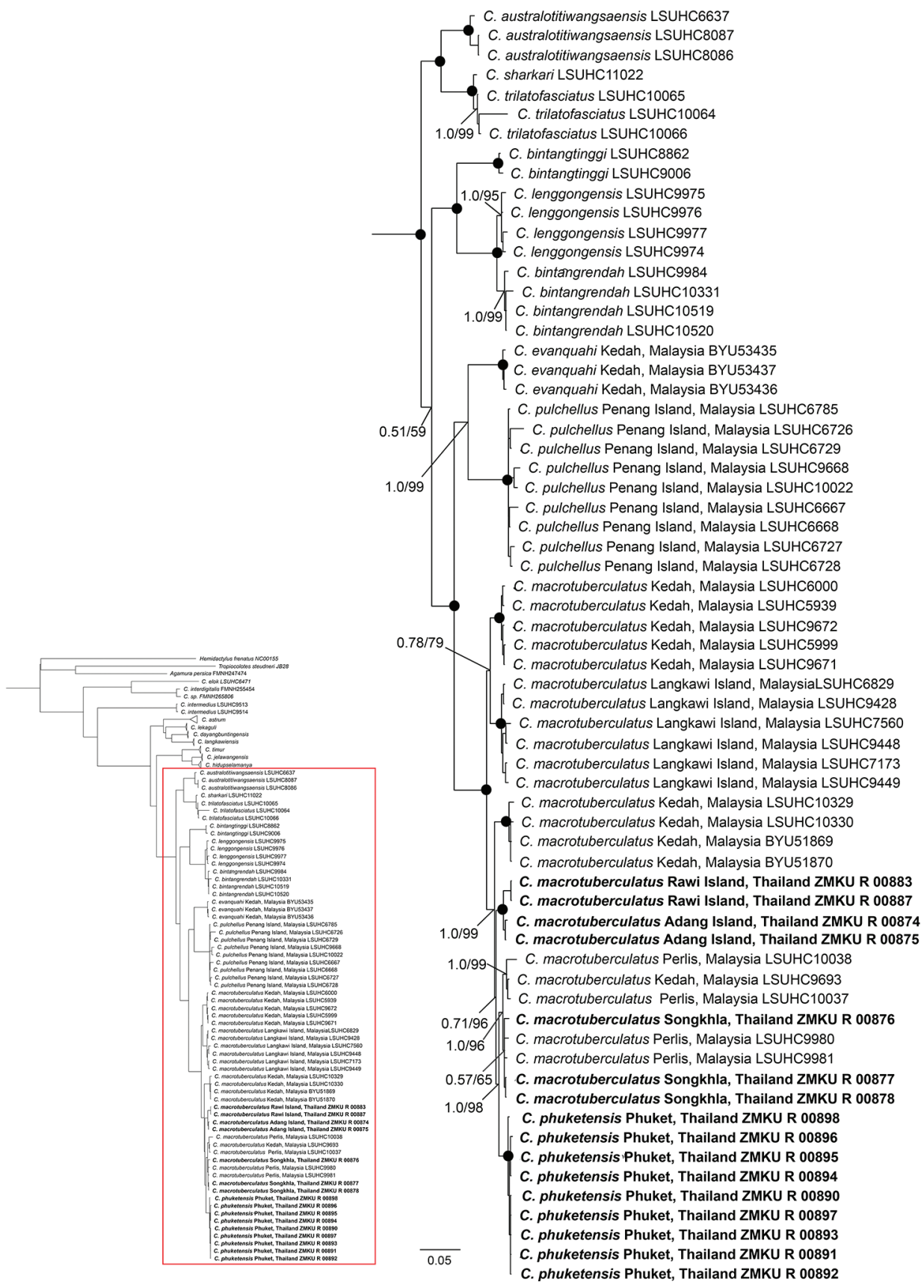

Figure 2. Reconstructed phylogenetic relationships of the Cyrtodactylus pulchellus complex based on 1,453 bp of ND2 and flanking tRNAs. The phylogenetic tree is from the Maximum Likelihood analysis with Bayesian posterior probabilities (BPP) and ultrafast bootstrap support values (UFB), respectively. Black circles represent nodes supported by BPP and UFB of 1.0 and 100. Samples in bold are new sequence from this study. 
Table 3. Summary statistics and factor loadings of the principal component analysis from morphological characters for males and females Cyrtodactylus macrotuberculatus, C. phuketensis, and C. pulchellus. Morphological character abbreviations are defined in the Materials and methods. / = data unavailable.

\begin{tabular}{|c|c|c|c|c|c|c|}
\hline \multirow[t]{2}{*}{ Characters } & \multicolumn{3}{|c|}{ Males } & \multicolumn{3}{|c|}{ Females } \\
\hline & PC1 & PC2 & PC3 & PC1 & PC2 & PC3 \\
\hline $\mathrm{SVL}_{\text {adj }}$ & 0.067 & -0.193 & -0.291 & 0.080 & -0.043 & -0.047 \\
\hline $\mathrm{TW}_{\mathrm{adj}}$ & 0.558 & 0.589 & -0.029 & -0.823 & 0.036 & 0.055 \\
\hline $\mathrm{FL}_{\mathrm{adj}}$ & 0.586 & -0.425 & 0.023 & -0.040 & 0.507 & -0.474 \\
\hline $\mathrm{TBL}_{\mathrm{adj}}$ & 0.467 & -0.690 & 0.027 & -0.055 & 0.452 & -0.752 \\
\hline $\mathrm{AG}_{\mathrm{adj}}$ & -0.185 & -0.442 & 0.415 & 0.685 & -0.284 & -0.283 \\
\hline $\mathrm{HL}_{\text {adj }}$ & 0.784 & 0.141 & -0.033 & 0.384 & 0.730 & 0.436 \\
\hline $\mathrm{HW}_{\mathrm{adj}}$ & 0.439 & 0.569 & -0.144 & 0.232 & 0.405 & -0.163 \\
\hline $\mathrm{HD}_{\text {adj }}$ & 0.577 & 0.539 & -0.221 & 0.516 & -0.070 & 0.040 \\
\hline $\mathrm{ED}_{\text {adj }}$ & 0.585 & -0.173 & 0.374 & -0.364 & 0.777 & 0.337 \\
\hline $\mathrm{EE}_{\text {adj }}$ & 0.484 & 0.314 & -0.580 & 0.581 & 0.338 & -0.056 \\
\hline $\mathrm{ES}_{\mathrm{adj}}$ & 0.858 & -0.053 & -0.017 & 0.669 & 0.260 & 0.455 \\
\hline $\mathrm{EN}_{\mathrm{adj}}$ & 0.774 & -0.289 & 0.004 & 0.726 & -0.087 & 0.133 \\
\hline $\mathrm{IO}_{\text {adj }}$ & 0.147 & 0.662 & 0.003 & 0.602 & 0.117 & 0.174 \\
\hline $\mathrm{EL}_{\text {adj }}$ & 0.351 & -0.097 & -0.360 & 0.141 & 0.190 & 0.714 \\
\hline $\mathrm{IN}_{\text {adj }}$ & 0.527 & 0.224 & -0.184 & -0.216 & 0.150 & -0.473 \\
\hline Supralabials & -0.217 & 0.622 & 0.270 & -0.070 & -0.745 & 0.307 \\
\hline Infralabials & 0.218 & 0.627 & 0.402 & -0.258 & -0.690 & 0.340 \\
\hline Paravertebral tubercles & -0.157 & 0.472 & 0.409 & -0.287 & -0.196 & -0.168 \\
\hline Longitudinal tubercles & 0.363 & 0.215 & 0.642 & -0.133 & 0.215 & 0.464 \\
\hline Ventral scales & 0.761 & -0.297 & 0.338 & -0.665 & 0.497 & 0.230 \\
\hline $4^{\text {th }}$ toe lamellae & 0.439 & -0.324 & -0.217 & -0.864 & 0.095 & 0.283 \\
\hline Femoroprecloacal pores & 0.512 & -0.196 & 0.468 & 1 & 1 & 1 \\
\hline Eigenvalue & 5.671 & 3.864 & 2.164 & 4.859 & 3.484 & 2.784 \\
\hline Percentage of variance & 25.776 & 17.562 & 9.834 & 23.136 & 16.592 & 13.258 \\
\hline Cumulative proportion & 25.776 & 43.338 & 53.172 & 23.136 & 39.728 & 52.986 \\
\hline
\end{tabular}

and Mann-Whitney $U$ tests, $p=0.1765-0.9523)$ except only $\mathrm{IO}_{\text {adj }}(t$-test, $p=0.0256)$. In adult females, $C$. macrotuberculatus $(N=11)$ and $C$. phuketensis $(N=4)$ were not significantly different in twelve morphological characters ( $t$-tests and Welch's $t$-test, $p=0.2325-0.9626)$ whereas only three characters were significantly different which are $\mathrm{TBL}_{\text {adj }}(t$-test, $p=0.0495), \mathrm{AG}_{\text {adj }}($ Mann-Whitney $\mathrm{U}$ tests, $p=0.0176)$ and $\mathrm{IN}_{\mathrm{adj}}$ (Welch's $t$-test, $p=0.0129$ ).

Sumontha et al. (2012) distinguished C. phuketensis from C. macrotuberculatus by using the number of subdigital lamellae on the fourth toe, number of dark body bands, and the presence of a precloacal groove in females. Based on examination of the type material and newly collected specimens of $C$. phuketensis from Phuket Island, these diagnostic characters overlap with those characters of $C$. macrotuberculatus. In this study, C. phuketensis has 19-21 total subdigital lamellae on the fourth toe (vs. 19-23 in C. macrotuberculatus); three or four dark body bands (vs. three or four in C. macrotuberculatus), and no precloacal groove in females (also absent in C. macrotuberculatus; Table 5). 

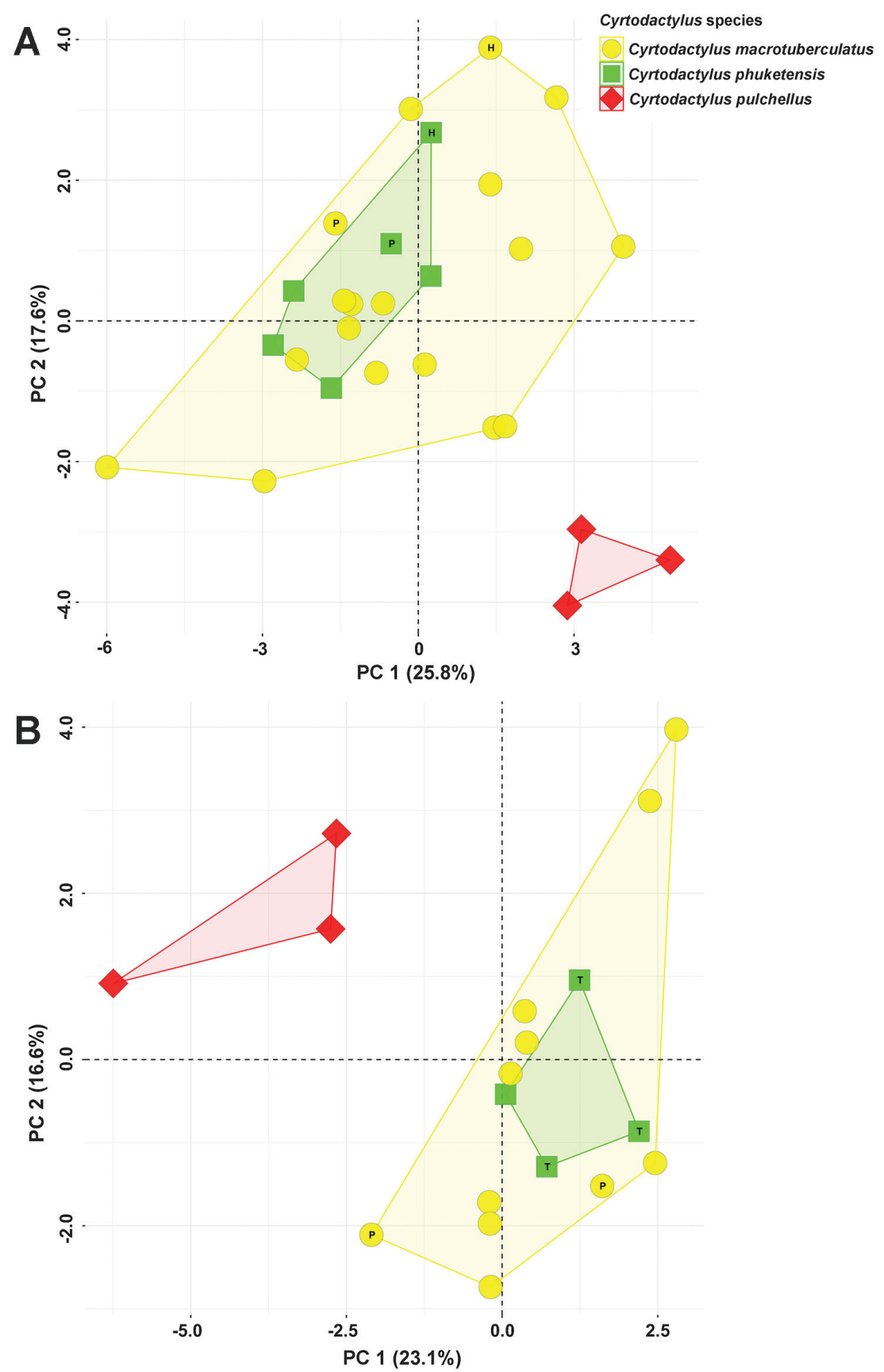

Figure 3. Plots for the first two principal components of morphological characters from $\mathbf{A}$ males, and B females resulting from the principal component analyses of Cyrtodactylus macrotuberculatus (yellow circles), C. phuketensis (green squares) and C. pulchellus (red diamonds). The letters in the scatter plots refer to holotype $(=\mathrm{H})$, paratype $(=\mathrm{P})$ and topotype $(=\mathrm{T})$. 
Table 4. Comparisons of fifteen morphological characters between Cyrtodactylus macrotuberculatus and C. phuketensis. Data are given as mean and standard deviation, followed by range in parentheses. Morphological character abbreviations are defined in the Materials and methods. Key: ${ }^{a}$ tested by Welch's $t$-test, ${ }^{b}$ tested by Mann-Whitney $\mathrm{U}$ test, ${ }^{*}$ significance level at $p<0.05$.

\begin{tabular}{|c|c|c|c|c|c|c|c|c|}
\hline \multirow[t]{3}{*}{ Characters } & \multicolumn{2}{|l|}{ Males } & \multicolumn{6}{|c|}{ Females } \\
\hline & C. macrotuberculatus & C.phuketensis & $t$-test & $p$ & C. macrotuberculatus & C.phuketensis & $t$-test & $p$ \\
\hline & $N=18$ & $N=6$ & & & $N=11$ & $N=4$ & & \\
\hline SVL & $\begin{array}{c}105.8 \pm 8.9 \\
(88.9-117.9)\end{array}$ & $\begin{array}{l}105.3 \pm 10.0 \\
(93.2-115.3)\end{array}$ & 0.112 & 0.9122 & $\begin{array}{l}103.7 \pm 10.1 \\
(84.1-115.7)\end{array}$ & $\begin{array}{l}104.4 \pm 16.0 \\
(84.8-117.6)\end{array}$ & -0.098 & 0.9237 \\
\hline TW & $\begin{array}{c}9.5 \pm 0.9 \\
(8.0-11.6)\end{array}$ & $\begin{array}{c}9.6 \pm 1.2 \\
(7.9-10.9)\end{array}$ & -0.061 & 0.9523 & $\begin{array}{c}8.1 \pm 1.4 \\
(5.9-10.3)\end{array}$ & $\begin{array}{c}8.2 \pm 2.1 \\
(6.2-10.5)\end{array}$ & -0.352 & 0.7307 \\
\hline FL & $\begin{array}{c}17.2 \pm 1.5 \\
(14.2-18.9)\end{array}$ & $\begin{array}{c}17.2 \pm 2.0 \\
(14.4-19.0)\end{array}$ & -0.098 & 0.9229 & $\begin{array}{c}16.8 \pm 1.6 \\
(13.6-18.3)\end{array}$ & $\begin{array}{c}16.8 \pm 2.7 \\
(13.3-19.3)\end{array}$ & 0.048 & 0.9626 \\
\hline TBL & $\begin{array}{c}20.3 \pm 1.6 \\
(17.3-22.8)\end{array}$ & $\begin{array}{c}20.6 \pm 2.6 \\
(17.4-19.0)\end{array}$ & -0.631 & 0.5348 & $\begin{array}{c}19.9 \pm 1.8 \\
(16.3-21.9)\end{array}$ & $\begin{array}{c}20.4 \pm 3.2 \\
(16.5-23.2)\end{array}$ & -2.166 & $0.0495^{*}$ \\
\hline AG & $\begin{array}{c}50.4 \pm 4.6 \\
(41.3-58.6)\end{array}$ & $\begin{array}{c}51.5 \pm 5.9 \\
(44.7-57.4)\end{array}$ & -1.034 & 0.3125 & $\begin{array}{c}50.7 \pm 4.5 \\
(42.0-56.0)\end{array}$ & $\begin{array}{c}53.4 \pm 7.9 \\
(43.4-60.2)\end{array}$ & $4^{\mathrm{b}}$ & $0.0176^{*}$ \\
\hline $\mathrm{HL}$ & $\begin{array}{c}29.4 \pm 2.2 \\
(24.6-33.3)\end{array}$ & $\begin{array}{l}29.2 \pm 2.7 \\
(25.6-31.7)\end{array}$ & 0.590 & 0.5613 & $\begin{array}{c}28.5 \pm 3.1 \\
(22.8-32.3)\end{array}$ & $\begin{array}{l}28.4 \pm 4.4 \\
(23.1-32.1)\end{array}$ & $0.575^{\mathrm{a}}$ & 0.5758 \\
\hline HW & $\begin{array}{c}20.0 \pm 1.8 \\
(16.7-22.9)\end{array}$ & $\begin{array}{c}19.8 \pm 2.7 \\
(16.3-22.5)\end{array}$ & 0.416 & 0.6816 & $\begin{array}{c}19.0 \pm 1.9 \\
(15.6-21.0)\end{array}$ & $\begin{array}{c}18.7 \pm 2.6 \\
(15.8-21.4)\end{array}$ & 0.815 & 0.4297 \\
\hline HD & $\begin{array}{l}12.0 \pm 1.3 \\
(9.7-14.1)\end{array}$ & $\begin{array}{l}12.2 \pm 1.9 \\
(9.9-14.2)\end{array}$ & -0.840 & 0.4100 & $\begin{array}{l}11.3 \pm 1.3 \\
(9.0-13.4)\end{array}$ & $\begin{array}{l}11.5 \pm 2.0 \\
(9.1-13.5)\end{array}$ & -1.054 & 0.3109 \\
\hline ED & $\begin{array}{l}6.9 \pm 0.6 \\
(5.8-7.9)\end{array}$ & $\begin{array}{l}6.7 \pm 0.5 \\
(5.6-7.0)\end{array}$ & 1.397 & 0.1765 & $\begin{array}{l}6.7 \pm 0.7 \\
(5.6-7.6)\end{array}$ & $\begin{array}{l}6.5 \pm 1.1 \\
(7.4-9.6)\end{array}$ & 1.253 & 0.2325 \\
\hline $\mathrm{EE}$ & $\begin{array}{l}8.6 \pm 1.0 \\
(6.5-9.8)\end{array}$ & $\begin{array}{l}8.6 \pm 1.0 \\
(7.1-9.4)\end{array}$ & $62^{\mathrm{b}}$ & 0.6261 & $\begin{array}{l}8.5 \pm 0.8 \\
(7.0-9.4)\end{array}$ & $\begin{array}{l}8.6 \pm 1.1 \\
(7.4-9.6)\end{array}$ & -0.106 & 0.9171 \\
\hline ES & $\begin{array}{c}11.7 \pm 1.0 \\
(10.0-13.6)\end{array}$ & $\begin{array}{c}11.7 \pm 1.1 \\
(10.3-12.9)\end{array}$ & $56^{\mathrm{b}}$ & 0.9225 & $\begin{array}{l}11.5 \pm 1.2 \\
(9.3-13.1)\end{array}$ & $\begin{array}{l}11.4 \pm 1.8 \\
(9.2-12.9)\end{array}$ & $0.271^{\mathrm{a}}$ & 0.7912 \\
\hline $\mathrm{EN}$ & $\begin{array}{l}8.7 \pm 0.7 \\
(7.3-9.8)\end{array}$ & $\begin{array}{l}8.7 \pm 0.7 \\
(7.6-9.5)\end{array}$ & 0.090 & 0.9288 & $\begin{array}{l}8.6 \pm 0.9 \\
(6.8-9.8)\end{array}$ & $\begin{array}{l}8.5 \pm 1.3 \\
(6.8-9.6)\end{array}$ & 0.610 & 0.5521 \\
\hline IO & $\begin{array}{l}5.1 \pm 0.6 \\
(4.0-6.3)\end{array}$ & $\begin{array}{l}4.8 \pm 0.6 \\
(4.1-5.5)\end{array}$ & 2.394 & $0.0256^{*}$ & $\begin{array}{l}4.8 \pm 0.8 \\
(3.4-5.7)\end{array}$ & $\begin{array}{l}4.6 \pm 0.9 \\
(3.6-5.5)\end{array}$ & 0.835 & 0.4186 \\
\hline EL & $\begin{array}{l}2.3 \pm 0.3 \\
(1.7-2.8)\end{array}$ & $\begin{array}{l}2.2 \pm 0.4 \\
(1.4-2.6)\end{array}$ & 0.504 & 0.6192 & $\begin{array}{l}2.4 \pm 0.4 \\
(1.6-3.0)\end{array}$ & $\begin{array}{l}2.2 \pm 0.5 \\
(1.5-2.5)\end{array}$ & 1.125 & 0.281 \\
\hline IN & $\begin{array}{l}2.2 \pm 0.3 \\
(1.7-2.7)\end{array}$ & $\begin{array}{l}2.3 \pm 0.3 \\
(1.7-2.5)\end{array}$ & -0.352 & 0.7282 & $\begin{array}{l}2.0 \pm 0.5 \\
(1.3-2.9)\end{array}$ & $\begin{array}{l}2.3 \pm 0.3 \\
(1.9-2.6)\end{array}$ & $-1.785^{a}$ & $0.0129^{*}$ \\
\hline
\end{tabular}

\section{Systematics}

The phylogenetic analyses recovered $C$. phuketensis as being nested within the $C$. macrotuberculatus and bearing a low genetic divergence (mean 2.63\%) which was similar to that within C. macrotuberculatus populations (mean $2.48 \%$ ). In concordance, the statistical analyses of meristic and mensural characters of $C$. phuketensis widely overlap with those of $C$. macrotuberculatus. Based on these data, we propose that $C$. phuketensis from Phuket Island, Phuket Province is a junior synonym of $C$. macrotuberculatus which can be recognized as follows.

\section{Taxonomy}

\section{Cyrtodactylus macrotuberculatus Grismer and Ahmad, 2008}

Figure 4

Cyrtodactylus macrotuberculatus Grismer \& Ahmad, 2008: 55; Grismer 2011: 406;

Grismer et al. 2012: 45.

Cyrtodactylus phuketensis Sumontha et al., 2012: 62. 
Table 5. Summarized diagnostic characters of Cyrtodactylus macrotuberculatus and C. phuketensis taken from original descriptions (Grismer and Ahmad 2008; Sumontha et al. 2012) and this study based on type materials and newly additional specimens. / = data unavailable.

\begin{tabular}{|c|c|c|c|c|}
\hline & C. macrotuberculatus & C. phuketensis & C. macrotuberculatus & C. phuketensis \\
\hline & $\begin{array}{l}\text { (Grismer and Ahmad, } \\
\text { 2008) }\end{array}$ & $\begin{array}{l}\text { (Sumontha et al., } \\
\text { 2012) }\end{array}$ & This study & This study \\
\hline Supralabials & $10-12$ & $12-13$ & $9-12$ & $9-13$ \\
\hline Infralabials & $8-11$ & $9-10$ & $7-11$ & $7-10$ \\
\hline Tuberculation & Prominent & Prominent & Prominent & Prominent \\
\hline Tubercles on ventral surface of forelimbs & Yes & Yes & Yes & Yes \\
\hline Tubercles in gular region & Yes & Yes & Yes & Yes \\
\hline Ventrolateral fold tuberculate & Yes & Yes & Yes & Yes \\
\hline Paravertebral tubercles & $40-47$ & $40-43$ & $34-49$ & $39-45$ \\
\hline Longitudinal rows of tubercles & $22-26$ & $23-24$ & $19-27$ & $20-24$ \\
\hline Ventral scales & $19-22$ & $22-24$ & $17-28$ & $20-24$ \\
\hline $4^{\text {th }}$ toe lamellae & $21-23$ & 19 & $19-23$ & $19-21$ \\
\hline Femoroprecloacal pores & $35-37$ & $33-36$ & $28-42$ & $31-33$ \\
\hline Precloacal groove present in females & No & Yes & No & No \\
\hline Precloacal depression in males & No & No & Deep & Deep \\
\hline No. of body bands & 4 & $\begin{array}{l}3 \text { ( } 3.5 \text { one } \\
\text { individual) }\end{array}$ & $3-4$ & $\begin{array}{c}3-4(3+1 \\
\text { incomplete band })\end{array}$ \\
\hline Body band/interspace ratio & l & 1 & $0.95-1.74$ & $1.02-1.50$ \\
\hline $\begin{array}{l}\text { Dorsum bearing scattered pattern of } \\
\text { white tubercles }\end{array}$ & No & No & No & No \\
\hline Hatchlings/juveniles with white tail tip & No & No & No & No \\
\hline Dark caudal bands on original tail & l & 8 & $7-10$ & $7-8$ \\
\hline White caudal bands in adults immaculate & l & I & No & No \\
\hline Maximum SVL & 120.0 & 114.7 & 117.87 & 117.61 \\
\hline Sample size & 5 & 3 & 29 & 10 \\
\hline
\end{tabular}

Type specimens. Holotype (adult male, ZRC 2.6754) from Malaysia, Kedah, Pulau Langkawi, Gunung Raya; Paratypes: Malaysia, Kedah, Pulau Langkawi, Gunung Raya: ZRC 2.6755-2.6756, Telaga Tujuh: ZRC 2.6757, Lubuk Semilang: ZRC 2.6758.

Additional specimens examined (including types of $\boldsymbol{C}$. phuketensis). MALAYSIA - Kedah, Pulau Langkawi, Gunung Raya: LSUHC 09428-09429, LSUHC 09432; Perlis, Perlis State Park: LSUHC 09981, LSUHC 10097, ZRC 2.4869. Thailand - Satun Province, Mueang Satun District, Adang Island: ZMKU R 00871-00875, ZMKU R 00879-00882, Rawi Island: ZMKU R 00883-00889; Songkhla Province, Hat Yai District, Chalung Sub-district: ZMKU R 00876-00878; Phuket Province, Thalang District: PSUZC-RT 2010.58, THNHM 15378, ZMKU R 00894-00896, Kathu District: ZMKU R 00890-00893, ZMKU R 00897-00898 (Table 1).

Expanded diagnosis. Cyrtodactylus macrotuberculatus can be separated from all other species of $C$. pulchellus complex by having the following combination of characters (Table 6): (1) maximum SVL $117.9 \mathrm{~mm}$ (mean 105.0 \pm SD 9.8, $N=39$ ); (2) 9-13 supralabial and 7-11 infralabial scales; (3) prominent tuberculation on body; (4) tubercles on ventral surface of forelimbs, gular region, in ventrolateral body folds, and anterior one-third of tail; (5) 34-49 paravertebral tubercles; (6) 19-27 longitudinal tubercle rows; (7) 17-28 ventral scales; (8) 19-23 subdigital lamellae on the fourth 


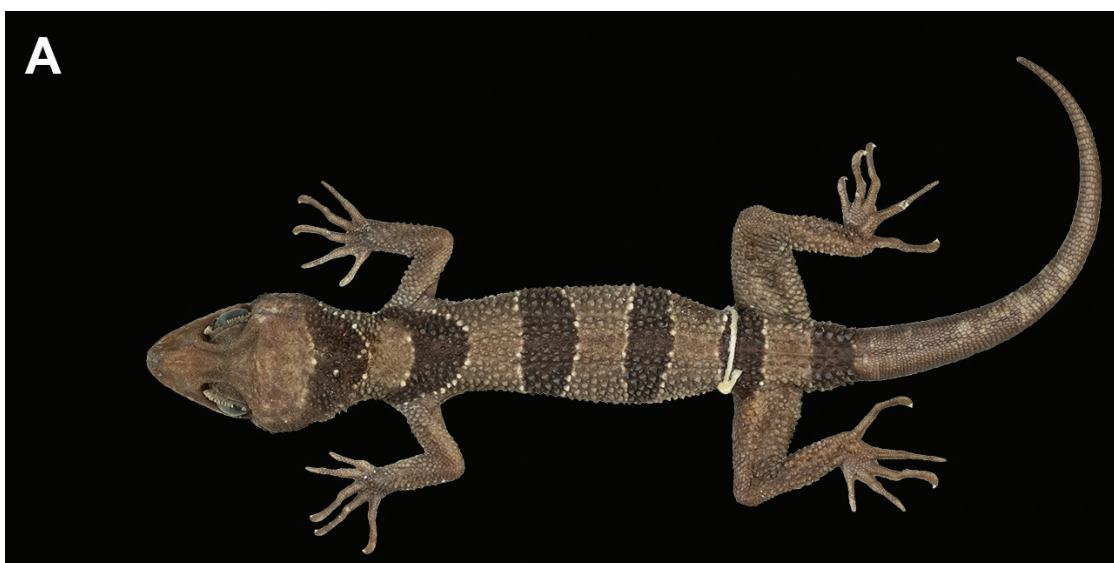

$\mathbf{B}$

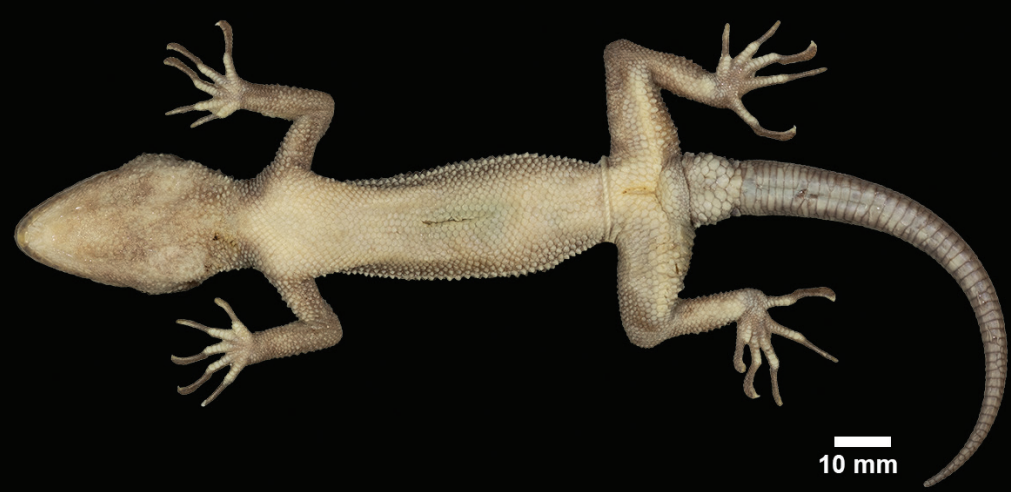

C

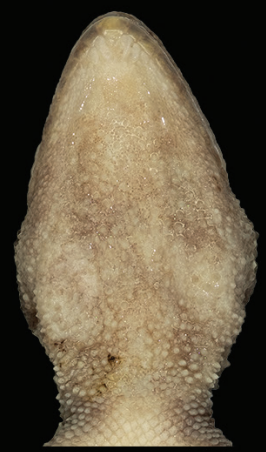

D

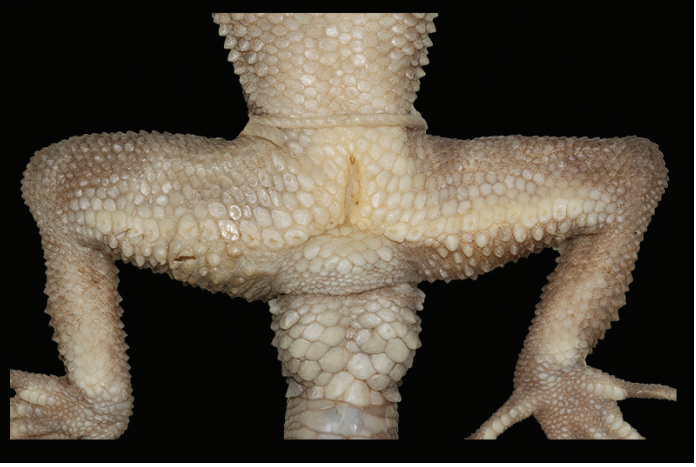

Figure 4. Male holotype of Cyrtodactylus macrotuberculatus from Pulau Langkawi, Kedah, Peninsular Malaysia (ZRC 2.6754) in preservative A dorsal view B ventral view $\mathbf{C}$ tuberculate gular region and throat, and $\mathbf{D}$ enlarge femoroprecloacal scale row and pores.

toe; (9) 28-42 femoroprecloacal pores in males; (10) deep precloacal groove in males; (11) three or four dark dorsal body bands; (12) body band wider than interspace; (13) 7-10 $(N=12)$ ringed dark caudal bands on original tail; (14) white caudal bands 
Table 6. Morphological measurement $(\mathrm{mm})$, meristic and non-meristic data from males and females of Cyrtodactylus macrotuberculatus. Morphological character abbreviations are defined in the Materials and methods.

\begin{tabular}{|c|c|c|c|c|c|c|}
\hline \multirow[t]{2}{*}{ Characters } & \multicolumn{2}{|c|}{ Adult males $(N=24)$} & \multicolumn{2}{|c|}{ Adult females $(N=15)$} & \multicolumn{2}{|c|}{ All $(N=39)$} \\
\hline & Mean \pm SD & (Min-Max) & Mean \pm SD & (Min-Max) & Mean \pm SD & (Min-Max) \\
\hline SVL & $105.7 \pm 9.0$ & $(88.9-117.9)$ & $103.9 \pm 11.3$ & $(84.1-117.6)$ & $105.0 \pm 9.8$ & $(84.1-117.9)$ \\
\hline TW & $9.5 \pm 1.0$ & $(7.9-11.6)$ & $8.2 \pm 1.5$ & $(5.9-10.5)$ & $9.0 \pm 1.4$ & $(5.9-11.6)$ \\
\hline FL & $17.2 \pm 1.6$ & $(14.2-19.0)$ & $16.8 \pm 1.8$ & $(13.3-19.3)$ & $17.1 \pm 1.7$ & $(13.3-19.3)$ \\
\hline TBL & $20.4 \pm 1.9$ & $(17.3-23.5)$ & $20.0 \pm 2.1$ & $(16.3-19.3)$ & $20.2 \pm 2.0$ & $(16.3-23.5)$ \\
\hline AG & $50.7 \pm 4.9$ & $(41.3-58.6)$ & $51.4 \pm 5.4$ & $(42.0-60.2)$ & $51.0 \pm 5.0$ & $(41.3-60.2)$ \\
\hline HL & $29.4 \pm 2.3$ & $(24.6-33.3)$ & $28.5 \pm 3.3$ & $(22.8-32.3)$ & $29.0 \pm 2.7$ & $(22.8-33.3)$ \\
\hline HW & $20.0 \pm 2.0$ & $(16.3-22.9)$ & $18.9 \pm 2.0$ & $(15.6-21.4)$ & $19.6 \pm 2.0$ & $(15.6-22.9)$ \\
\hline HD & $12.1 \pm 1.4$ & $(9.7-14.2)$ & $11.3 \pm 1.4$ & $(9.0-13.5)$ & $11.8 \pm 1.4$ & $(9.0-14.2)$ \\
\hline ED & $6.8 \pm 0.6$ & $(5.6-7.9)$ & $6.7 \pm 0.8$ & $(5.2-7.5)$ & $6.8 \pm 0.7$ & $(5.2-7.9)$ \\
\hline $\mathrm{EE}$ & $8.6 \pm 1.0$ & $(6.5-9.8)$ & $8.5 \pm 0.9$ & $(7.0-9.6)$ & $8.6 \pm 0.7$ & $(5.2-7.9)$ \\
\hline ES & $11.7 \pm 1.0$ & $(10.0-13.6)$ & $11.5 \pm 1.3$ & $(9.2-13.1)$ & $11.6 \pm 1.1$ & $(9.2-13.6)$ \\
\hline EN & $8.7 \pm 0.7$ & $(7.3-9.8)$ & $8.6 \pm 1.0$ & $(6.8-9.8)$ & $8.6 \pm 0.8$ & $(6.8-9.8)$ \\
\hline IO & $5.0 \pm 0.6$ & $(4.0-6.3)$ & $4.8 \pm 0.8$ & $(3.4-5.7)$ & $4.8 \pm 0.7$ & $(3.4-6.3)$ \\
\hline EL & $2.3 \pm 0.3$ & $(1.4-2.8)$ & $2.3 \pm 0.4$ & $(1.5-3.0)$ & $2.3 \pm 0.4$ & $(1.4-3.0)$ \\
\hline IN & $2.2 \pm 0.5$ & $(1.7-2.7)$ & $2.1 \pm 0.5$ & $(1.3-2.9)$ & $2.2 \pm 0.4$ & $(1.3-2.9)$ \\
\hline HL/SVL & $0.28 \pm 0.01$ & $(0.27-0.30)$ & $0.27 \pm 0.01$ & $(0.26-0.29)$ & $0.28 \pm 0.01$ & $(0.26-0.30)$ \\
\hline $\mathrm{HW} / \mathrm{HL}$ & $0.68 \pm 0.03$ & $(0.62-0.74)$ & $0.67 \pm 0.02$ & $(0.62-0.70)$ & $0.67 \pm 0.03$ & $(0.62-0.74)$ \\
\hline $\mathrm{HD} / \mathrm{HL}$ & $0.41 \pm 0.02$ & $(0.37-0.45)$ & $0.40 \pm 0.01$ & $(0.38-0.42)$ & $0.41 \pm 0.02$ & $(0.37-0.45)$ \\
\hline ES/HL & $0.40 \pm 0.01$ & $(0.37-0.41)$ & $0.40 \pm 0.00$ & $(0.39-0.41)$ & $0.40 \pm 0.01$ & $(0.37-0.41)$ \\
\hline $\mathrm{ED} / \mathrm{HL}$ & $0.23 \pm 0.01$ & $(0.21-0.27)$ & $0.23 \pm 0.01$ & $(0.22-0.25)$ & $0.23 \pm 0.01$ & $(0.21-0.27)$ \\
\hline EL/HL & $0.08 \pm 0.01$ & $(0.05-0.10)$ & $0.08 \pm 0.01$ & $(0.05-0.10)$ & $0.08 \pm 0.01$ & $(0.05-0.10)$ \\
\hline AG/SVL & $0.48 \pm 0.02$ & $(0.43-0.51)$ & $0.50 \pm 0.01$ & $(0.47-0.52)$ & $0.49 \pm 0.02$ & $(0.43-0.52)$ \\
\hline FL/SVL & $0.16 \pm 0.00$ & $(0.15-0.17)$ & $0.16 \pm 0.00$ & $(0.16-0.17)$ & $0.16 \pm 0.00$ & $(0.15-0.17)$ \\
\hline TBL/SVL & $0.19 \pm 0.01$ & $(0.18-0.21)$ & $0.19 \pm 0.00$ & $(0.18-0.20)$ & $0.19 \pm 0.01$ & $(0.18-0.21)$ \\
\hline TL/SVL & $1.29 \pm 0.04$ & $(1.23-1.35)$ & $1.27 \pm 0.04$ & $(1.24-1.34)$ & $1.28 \pm 0.04$ & $(1.23-1.35)$ \\
\hline Supralabials & \multicolumn{2}{|c|}{$9-13$} & \multicolumn{2}{|c|}{$9-12$} & \multicolumn{2}{|c|}{$9-13$} \\
\hline Infralabials & \multicolumn{2}{|c|}{$7-11$} & \multicolumn{2}{|c|}{$7-11$} & \multicolumn{2}{|c|}{$7-11$} \\
\hline Tuberculation & \multicolumn{2}{|c|}{ Prominent } & \multicolumn{2}{|c|}{ Prominent } & \multicolumn{2}{|c|}{ Prominent } \\
\hline $\begin{array}{l}\text { Tubercles on ventral surface of } \\
\text { forelimbs }\end{array}$ & \multicolumn{2}{|c|}{ Yes } & \multicolumn{2}{|c|}{ Yes } & \multicolumn{2}{|c|}{ Yes } \\
\hline Tubercles in gular region & \multicolumn{2}{|c|}{ Yes } & \multicolumn{2}{|c|}{ Yes } & \multicolumn{2}{|c|}{ Yes } \\
\hline Ventrolateral fold tuberculate & \multicolumn{2}{|c|}{ Yes } & \multicolumn{2}{|c|}{ Yes } & \multicolumn{2}{|c|}{ Yes } \\
\hline Paravertebral tubercles & \multicolumn{2}{|c|}{$37-49$} & \multicolumn{2}{|c|}{$34-47$} & \multicolumn{2}{|c|}{$34-49$} \\
\hline Longitudinal rows of tubercles & & -27 & & -26 & & -27 \\
\hline Ventral scales & & -28 & & -26 & & -28 \\
\hline $4^{\text {th }}$ toe lamellae & & -23 & & -23 & & -23 \\
\hline Femoroprecloacal pores & & -42 & & No & & -42 \\
\hline Precloacal depression & & Tes & & No & Only & n males \\
\hline No. of body bands & & r 4 & & or 4 & & or 4 \\
\hline Body band/interspace ratio & 0.9 & -1.75 & 1.0 & -1.62 & 0.95 & -1.74 \\
\hline Dark caudal bands on original tail & & -9 & & -10 & & -10 \\
\hline
\end{tabular}

infused with dark pigmentation in adults; (15) posterior portion of tail in hatchlings and juveniles bands not white.

Description of adult males. SVL of adult males range from 88.9-117.9 mm (mean 105.7, $N=24$ ); head moderate in length (HL/SVL 0.27-0.30), width (HW/HL 0.62-0.74), somewhat flattened (HD/HL 0.37-0.45), distinct from neck, triangular in dorsal profile; lores concave; frontal and prefrontal regions deeply concave; canthus 
rostralis sharply rounded; snout elongate (ES/HL $0.37-0.41$ ), rounded in dorsal profile, laterally constricted; eye large (ED/HL 0.21-0.27); ear opening elliptical, moderate in size (EL/HL 0.05-0.10) obliquely oriented; eye to ear distance greater than diameter of eye; rostral rectangular, divided dorsally by an inverted $\mathrm{Y}$ or I-shaped furrow, bordered posteriorly by large left and right supranasals and small internasal, bordered laterally by external nares and first supralabials; external nares bordered anteriorly by rostral, dorsally by one large anterior supranasal, posteriorly by two postnasals, ventrally by first supralabial; 9-13 rectangular supralabials extending to just beyond upturn of labial margin, tapering abruptly below midpoint of eye; 7-11 infralabials not tapering in size posteriorly; scales of rostrum and lores slightly raised, larger than granular scales on top of head and occiput, those on posterior portion of canthus rostralis slightly larger; scales on top of head and occiput intermixed with enlarged tubercles; large, boney frontal ridges bordering orbit confluent with boney, transverse, parietal ridge; dorsal superciliaries elongate, smooth, largest anteriorly; mental triangular, bordered laterally by first infralabials and posteriorly by left and right trapezoidal postmentals that contact medially for $40-50 \%$ of their length posterior to mental; single row of slightly enlarged, elongate sublabials extending posteriorly to $5^{\text {th }}-7^{\text {th }}$ infralabial; small, granular, gular scales intermixed with numerous large, conical tubercles grading posteriorly into larger, conical tubercles on throat which abruptly transition into large, flat, smooth, imbricate, pectoral and ventral scales.

Body relatively short (AG/SVL 0.43-0.51) with well-defined, tuberculate, ventrolateral folds; dorsal scales small, granular, interspersed with large, trihedral, regularly arranged, keeled tubercles separated by no more than three granules at their base; tubercles extend from top of head onto approximately one-half of tail but not onto regenerated tail; tubercles on occiput and nape relatively small, those on body largest; approximately 19-27 longitudinal rows of dorsal tubercles at the mid body; approximately 37-49 paravertebral tubercles; $17-28$ flat, imbricate, ventral scales and much larger than dorsal scales; precloacal scales large, smooth; deep precloacal groove (= depression).

Forelimbs moderate in stature, relatively short (FL/SVL $0.15-0.17$ ); virtually no granular scales on dorsal surface of forelimbs, only large, trihedral, keeled tubercles; palmar scales slightly rounded; digits well-developed, inflected at basal, interphalangeal joints; subdigital lamellae nearly square proximal to joint inflection, only slightly expanded distal to inflection; digits more narrow distal to joints; claws well-developed, sheathed by dorsal and ventral scale; hind limbs more robust than forelimbs, moderate in length (TBL/SVL 0.18-0.21), virtually no granular scales on dorsal surfaces of hind limbs, only large, trihedral, keeled tubercles; ventral scales of thigh flat, smooth, imbricate; ventral, tibial scales flat, imbricate, slightly keeled; two rows of enlarged, flat, imbricate, femoroprecloacal scales extend from knee to knee through precloacal region where they are continuous with enlarged, pore-bearing precloacal scales; 2842 contiguous, pore-bearing femoroprecloacal scales forming an inverted $\mathrm{T}$ bearing a deep, precloacal groove; eight to eleven pores bordering groove; postfemoral scales immediately posterior to the pore-bearing scale row conical, forming an abrupt union 
on posteroventral margin of thigh; plantar scales low, slightly rounded; digits welldeveloped, inflected at basal, interphalangeal joints; subdigital lamellae proximal to joint inflection nearly square, only slightly expanded distal to inflection; digits more narrow distal to joints; claws well-developed, sheathed by a dorsal and ventral scale; 19-23 subdigital lamellae on the $4^{\text {th }}$ toe.

Original tail (TL/SVL) moderate in proportions, 123-135\% of SVL (mean 128, $N=12$ ), $7.9-11.6 \mathrm{~mm}$ in width at base, tapering to a point; dorsal scales at base of tail square, smooth, flat, subimbricate, lacking tubercle on regenerated tail; median row of transversely enlarged, subcaudal scales; shallow caudal furrow; two to five small, postcloacal tubercles at base of tail on hemipenial swellings; all postcloacal scales flat, large, imbricate.

Coloration of adult male ZMKU R 00871 in life (Fig. 5). Ground color of head, body, limbs, and dorsum light-brown to yellowish brown; wide, dark-brown nuchal band edged anteriorly and posteriorly by thin, creamy-white lines bearing tubercles extends from posterior margin of one eye to posterior margin of other eye; four similar body bands between nuchal loop and hind limb insertions edged anteriorly and posteriorly by thin, creamy-white lines bearing tubercles, first band terminates at shoulders, second and third bands terminate just dorsal to ventrolateral folds, the fourth band terminates at femurs; dark body bands slightly larger than light-colored interspaces; one additional dark-brown band posterior to hind limbs; original portion of tail bearing eight ringed, dark-colored bands separated by seven, narrower, off-white bands infused with dark pigmentation; ventral surfaces of head smudged with brown; abdomen and limbs beige, slightly darker, lateral regions.

Coloration in preservative (Fig. 6). Color pattern of head, body, limbs, and tail similar to that in life with some fading. Ground color of head, body, limbs, and dorsum tan; dark body and dark caudal bands lighter than in life.

Variation. Cyrtodactylus macrotuberculatus usually varies in coloration and banding pattern (Figs 7-8). In females, a precloacal groove and pores are absent (Fig. 9). PSUZC-RT 2010.58 and THNHM 15378 have a shallow precloacal groove. Three dark body bands occur in PSU 2010.58, THNHM 15378, ZMKU R 00889-00894 and ZMKU R 00897. In ZMKU R 00887, the second dorsal band bifurcates just dorsal to the ventrolateral fold. ZMKU R 00895 has four bands and the third band is incomplete. The third body band in ZMKU R 00896 is broken on the left of the midline and contacts the fourth body band bilaterally. Nuchal loop and body bands of ZMKU R 00883, ZMKU R 00895, and ZMKU R 00898 edged anteriorly and posteriorly by thin, light-yellow lines and tubercles; and dorsal superciliaries are light-yellow (Fig. 8). Variation in morphometric and meristic data are shown in Table 6.

Distribution. Cyrtodactylus macrotuberculatus is distributed on the mainland and only known from one island in Peninsular Malaysia and southern Thailand (Fig. 1). This species is known from Pulau Langkawi (Gunung Raya, Telaga Tujuh, Gunung Machinchang, and Lubuk Semilang), Kedah, Peninsular Malaysia (Grismer and Ahmad 2008). Other populations are found from Peninsular Malaysia; Kedah (Bukit Wang, Gunung Jerai, Hutan Lipur Sungai Tupah, Kuala Nerang, and Ulu Muda) and 


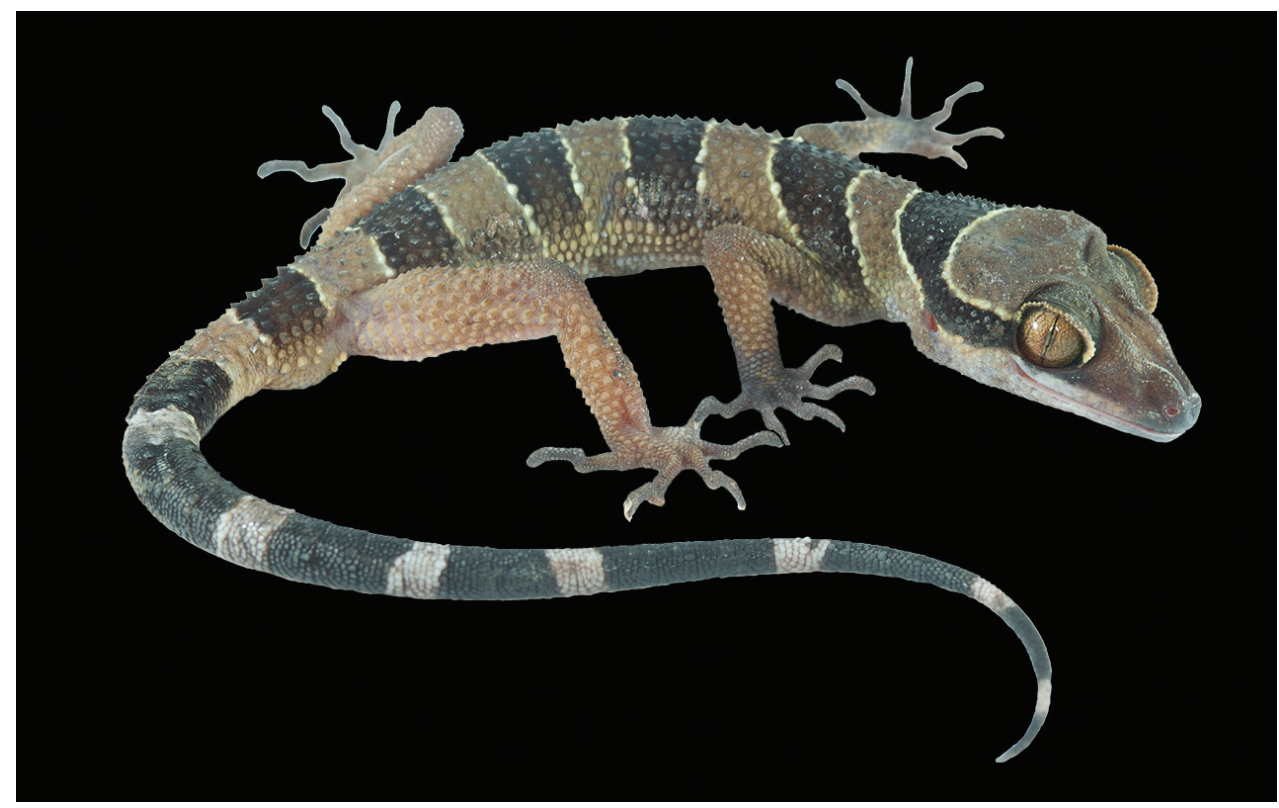

Figure 5. Adult male Cyrtodactylus macrotuberculatus from Adang Island, Satun Province, Thailand (ZMKU R 00871) in life.

Perlis (Bukit Chabang, Chuping and Perlis State Park; [Grismer 2011; Grismer et al. 2012; Quah et al. 2019]). In Thailand, C. macrotuberculatus was recorded from Phatthalung Province (Grismer et al. 2012); Phuket Province, Kathu District (Kathu Waterfall) and Thalang District (Thep Krasatti Sub-district, previously type locality of C. phuketensis); Satun Province, La-ngu District, and Mueang Satun District (Adang and Rawi Islands); Songkhla Province, Rattaphum District (Grismer et al. 2012) and Hat Yai District (Ton Nga Chang Waterfall).

Natural history. Based on specimens in Thailand, all individuals were found in similar habitat type, lowland forest habitat along granitic rock streams and surrounding areas (elevation 7-186 m asl) during a night survey (1900-2200; Fig. 10). The geckos were found mostly on rock boulders, vegetation (trunk of tree, buttress root, rotting wood and vines), and sometimes on the ground with leaf litter and high humidity (26.3-30.8 ${ }^{\circ} \mathrm{C}$ in temperature, $73.8-100 \%$ in relative humidity). Gravid female (ZMKU R 00876) contained four eggs during December. One juvenile (ZMKU $\mathrm{R} 00898,56.50 \mathrm{~mm}$ in SVL) was found on a tree trunk in January. The varied microhabitats within which this species occurs, are consistent with its characterization as a habitat generalist (Grismer et al. 2020, 2021b) and may account for its wide peninsular and insular distribution relative to other species of the pulchellus group whose distributions are much less extensive or site-specific (Grismer et al. 2012, 2014, 2016; Quah et al. 2019; Wood et al. 2020).

In Thailand, C. macrotuberculatus were found sympatric with other gecko species, Cnemaspis adangrawi Ampai et al., 2019 on Adang and Rawi Islands, Satun Province 


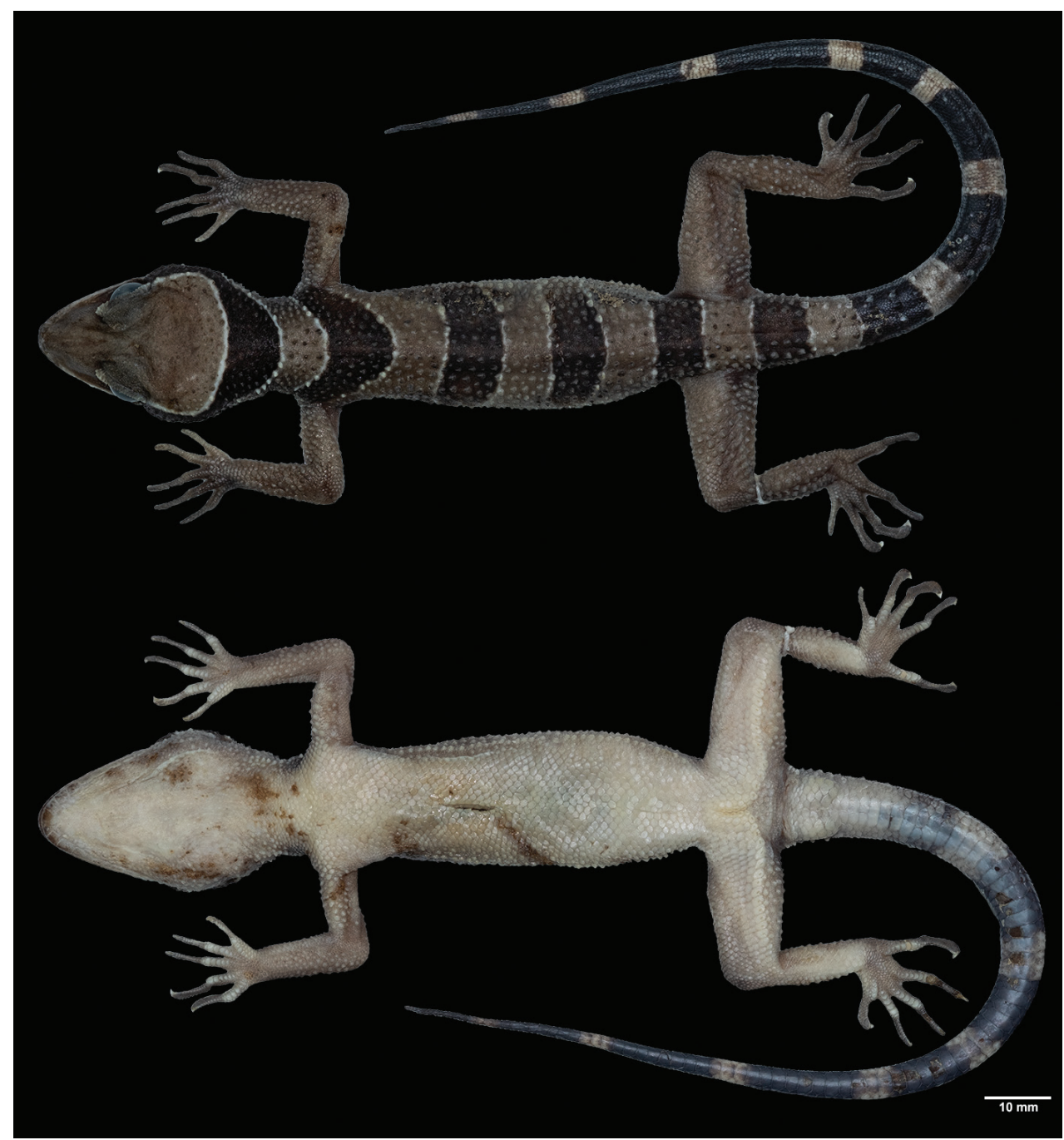

Figure 6. Adult male Cyrtodactylus macrotuberculatus from Adang Island, Satun Province, Thailand (ZMKU R 00871) in preservative $\mathbf{A}$ dorsal and $\mathbf{B}$ ventral views.

(Ampai et al. 2019); Cnemaspis phuketensis Das and Leong, 2004, Cyrtodactylus oldha$m i$ Theobald, 1876, and Gekko (Ptychozoon) tokehos Grismer et al., 2019 at Kathu and Thalang District, Phuket Province; G. (P.) tokehos, Cnemaspis kumpoli Taylor, 1963, and Gehyra mutilata (Weigmann, 1834) at Hat Yai District, Songkhla Province.

Comparison. Cyrtodactylus macrotuberculatus is distinguished from all other 15 species in the C. pulchellus complex by a combination of morphological characters (Table 7). It differs from all other species by having prominent tuberculation on the body; tubercles on ventral surface of forelimbs, gular region, and in ventrolateral body folds; 34-49 paravertebral tubercles; 19-27 longitudinal tubercle rows; 17-28 ventral scales; 


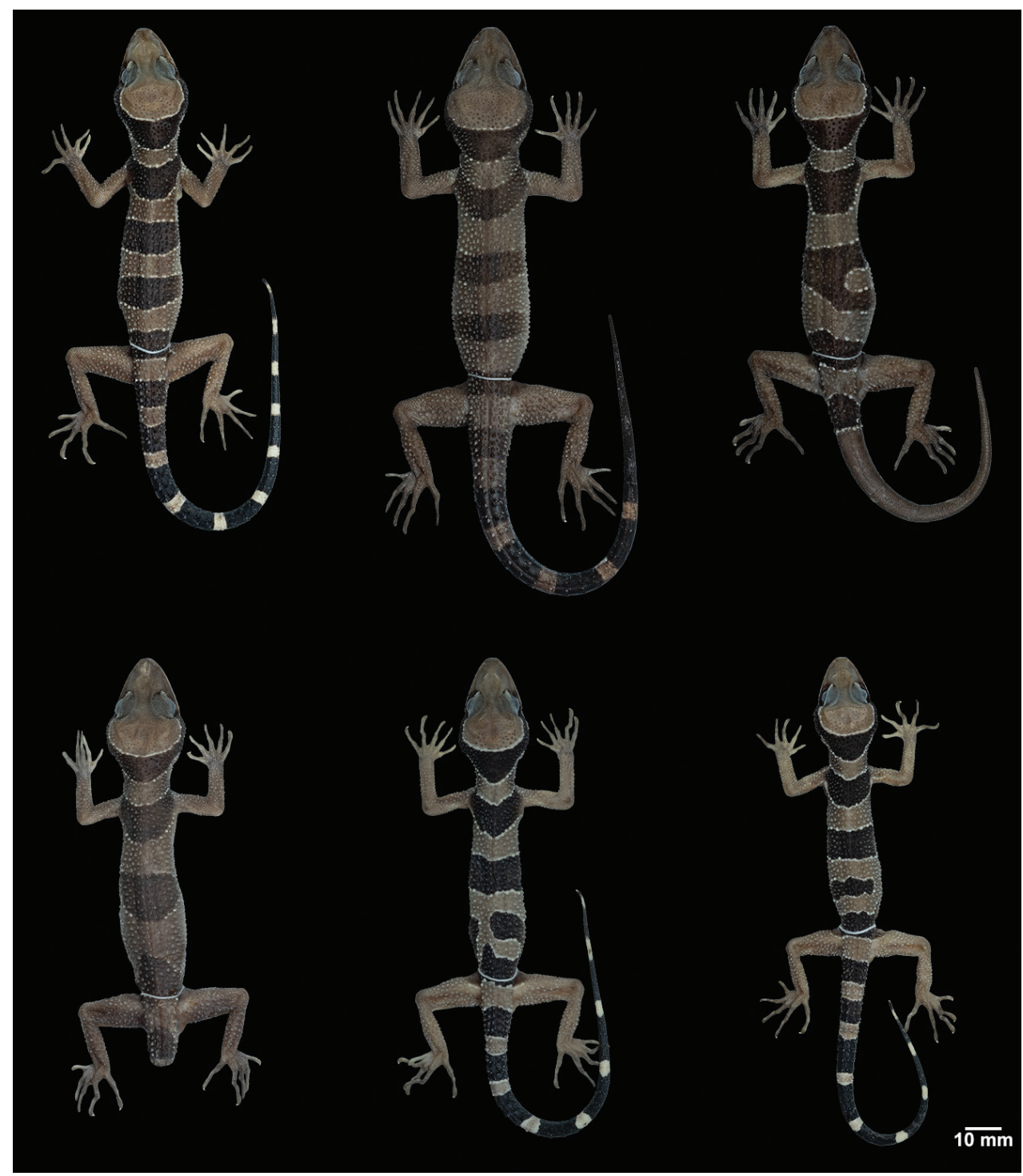

Figure 7. Variation in dorsal body band pattern of Cyrtodactylus macrotuberculatus from Thailand. From left to right, upper: ZMKU R 00878, ZMKU R 00873 from Adang Island, Satun Province; and ZMKU R 00887 from Rawi Island, Satun Province. Lower: ZMKU R 00889 from Rawi Island, Satun Province; ZMKU R 00896 and ZMKU R 00895 from Phuket Province.

19-23 subdigital lamellae on the fourth toe; $28-42$ femorprecloacal pores in males; deep precloacal groove in males; no scattered white spots on dorsum; 7-10 dark-ringed caudal bands on original tail; white caudal bands on original tail infused with dark pigmentation in adults. Additional comparisons between C. macrotuberculatus and other species in C. pulchellus complex are in Table 7. 


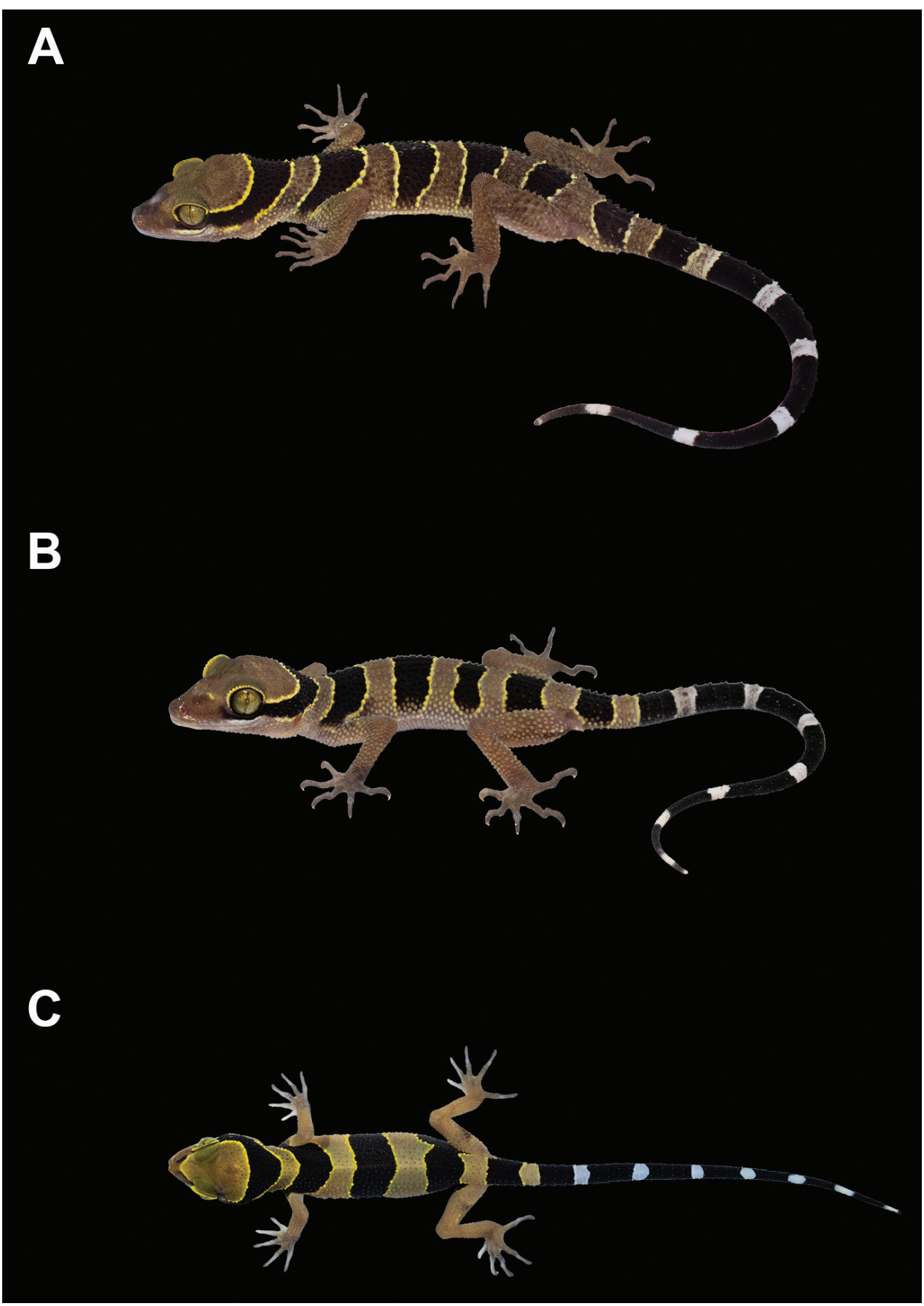

Figure 8. Color in life of Cyrtodactylus macrotuberculatus from Thailand $\mathbf{A}$ adult male ZMKU R 00883 from Rawi Island, Satun Province B subadult female ZMKU R 00895 from Thalang District, Phuket Province, and C juvenile ZMKU R 00898 from Kathu District, Phuket Province. 


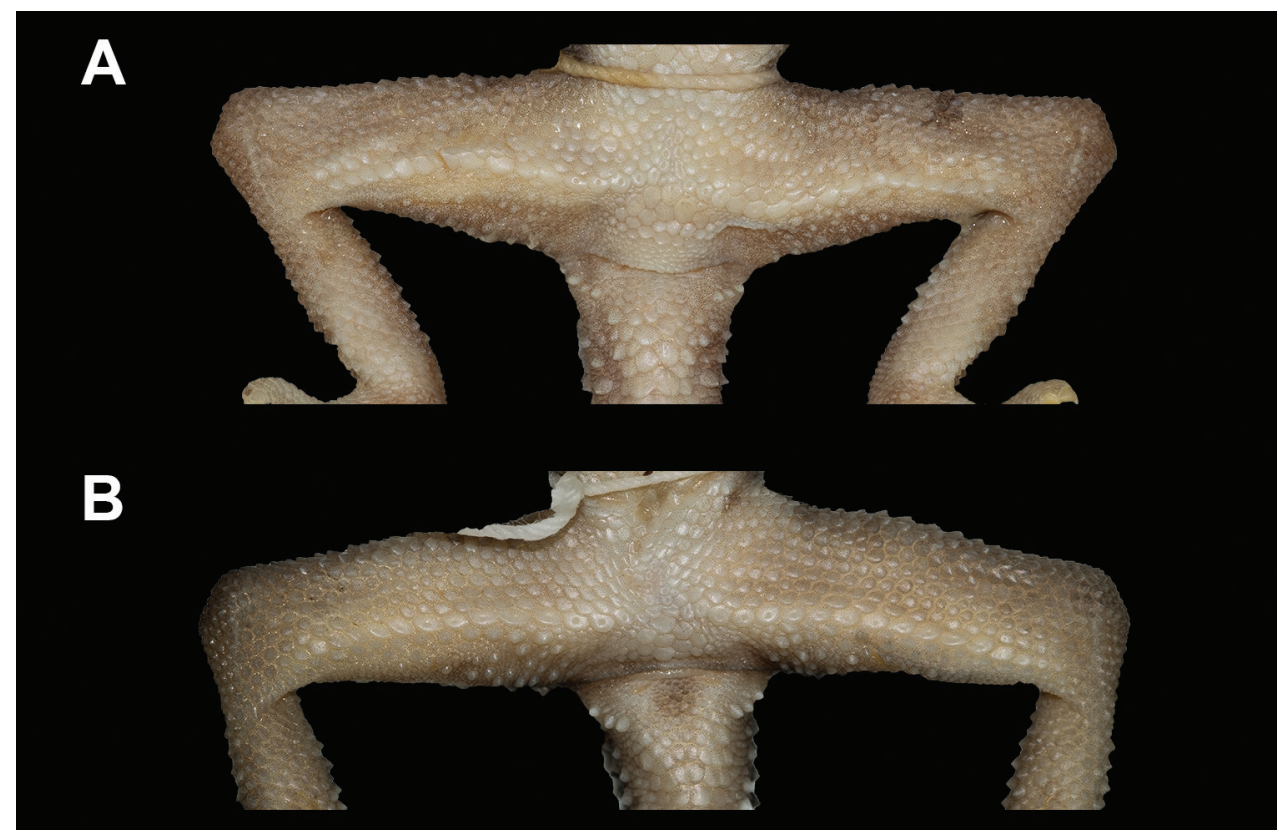

Figure 9. Precloacal region in female specimens of Cyrtodactylus macrotuberculatus A paratype ZRC 2.6758, from Telaga Tujuh, Pulau Langkawi, Malaysia, and B ZMKU R 00896 from Thalang District, Phuket Province, Thailand.

Based on molecular data, C. macrotuberculatus is the sister lineage to a clade composed of $C$. pulchellus and C. evanquahi. It can be separated from those two species by having tubercles on ventral surface of forelimbs, gular region, and in ventrolateral body folds (vs. absent in C. evanquahi and C. pulchellus); 17-28 ventral scales (vs. 29-33 in C. evanquahi and 29-34 in C. pulchellus); deep precloacal groove in males (vs. a shallow in C. evanquahi); three or four dark dorsal bands (vs. six or seven bands in $C$. evanquahi and only four bands in C. pulchellus); white posterior caudal region absent (vs. present in C. evanquahi); hatchlings and juveniles without white tail tip (vs. present in C. evanquahi).

\section{Discussion}

Cyrtodactylus macrotuberculatus and C. phuketensis are considered to be conspecific with the latter restricted to Phuket Island whereas C. macrotuberculatus is found on the Thai-Malay Peninsula and adjacent islands. The distinct characteristics between these two species were based solely on morphological comparisons by Sumontha et al. (2012). Our study provided additional morphology and molecular evidence to reassess the taxonomic status of $C$. macrotuberculatus and C. phuketensis from Thai populations and determine that these closely related populations are conspecific. Phylogenetic 


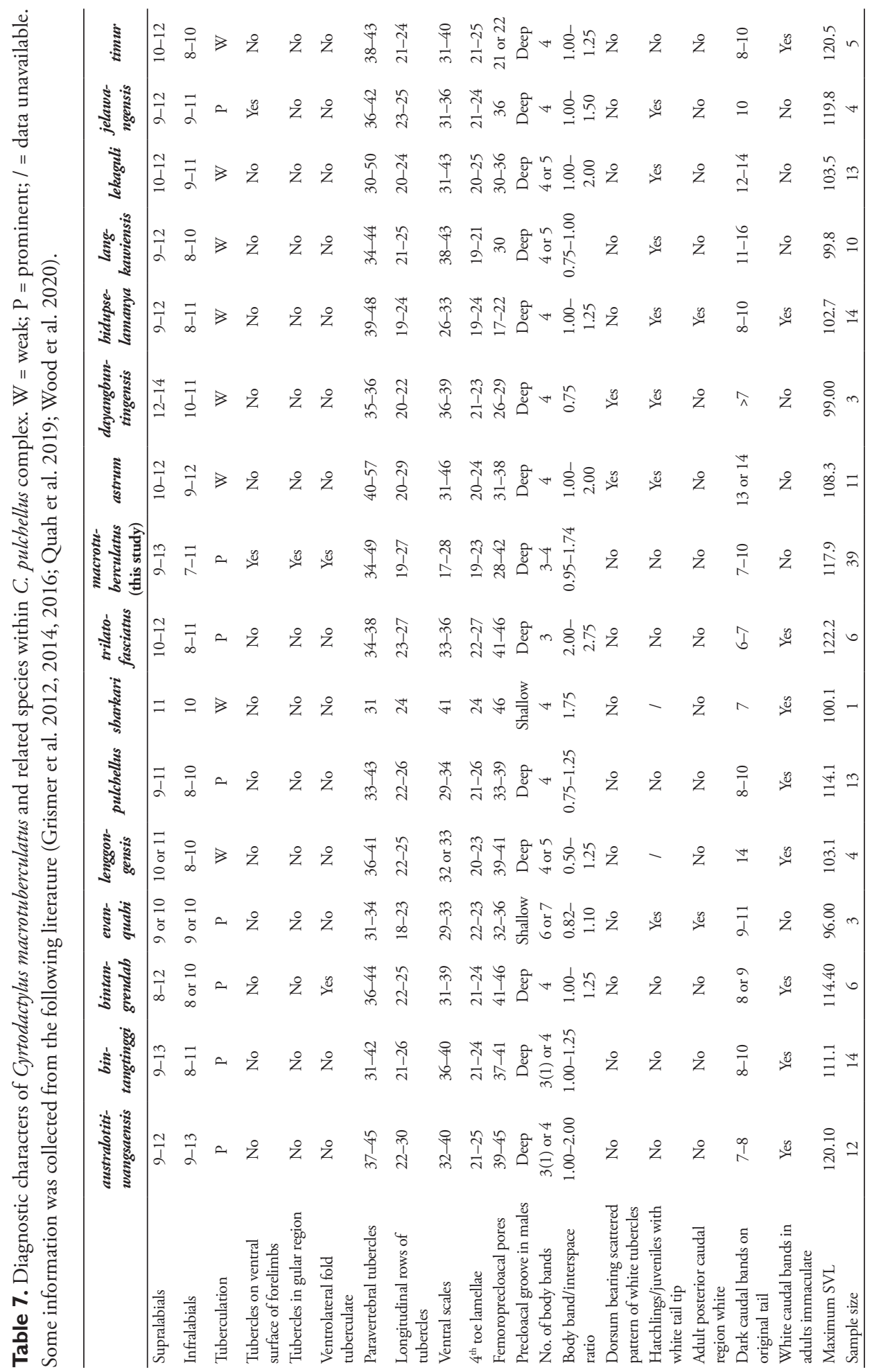




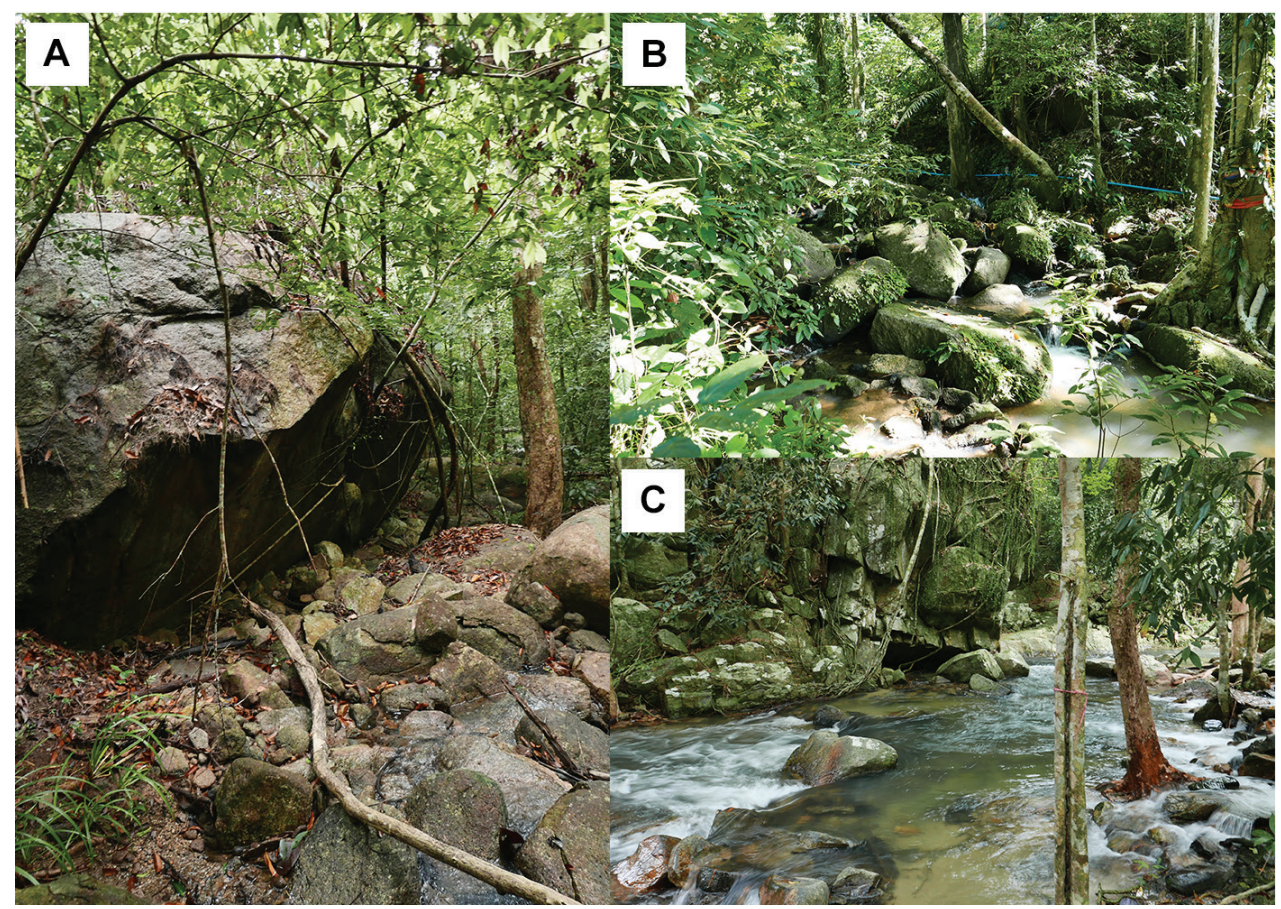

Figure 10. Habitats of Cyrtodactylus macrotuberculatus in Thailand A Adang Island, Satun Province B Kathu District, Phuket Province, and C Hat Yai District, Songkhla Province.

analyses from this study are concordant with the phylogenetic studies of Grismer et al. (2012, 2014, 2016), Quah et al. (2019), and Wood et al. (2020). Based on the dataset of ND2 gene and its flanking tRNA, the phylogenetic analyses recovered a clade of C. macrotuberculatus - including C. phuketensis - as a strongly supported monophyletic group consisting of multiple insular populations. Some substructuring occurs within the C. macrotuberculatus which could be the result of limited gene flow among isolated populations (Hurston et al. 2009; Jang et al. 2011) or local adaptation to different selection pressures in widely distributed habitat generalist.

Sumontha et al. (2012) diagnosed C. phuketensis by the number of bands between the limb insertions and the presence of a precloacal groove in the female paratype. We re-examined the type series of both species (except female paratype of C. phuketensis, QSMI 1170) and newly collected specimens. Variation in the number of bands was found in both species, similar to several species of the $C$. pulchellus group such as $C$. bintangrendah, C. australotitiwangsaensis and C. lenggongensis (Grismer et al. 2012, 2016).

Within the $C$. pulchellus group, a continuous series of enlarged femoroprecloacal scales forming an inverted $\mathrm{T}$ in the precloacal region is present in both sexes; however, the precloacal groove was found only in males. In the present study, the newly collected female specimens from the type locality of $C$. phuketensis had a continuous series of enlarged femoroprecloacal scales but lacked a precloacal groove (or depression) (Fig. 9). Therefore, this character is the same as in $C$. macrotuberculatus and all other species in the $C$. pulchellus group. The presence of a precloacal groove in the female specimen 
of C. phuketensis examined in Sumontha et al. (2012) was an erroneous observation (fig. 4 in Sumontha et al. 2012). The absence of a precloacal depression was used as a diagnostic character separating C. macrotuberculatus from C. phuketensis (see Grismer and Ahmad 2008; Sumontha et al. 2012). Based on the terminology of the precloacal depression in Mecke et al. (2016), the described specimens were re-examined and the presence of a precloacal depression (as precloacal groove) was observed in both C. macrotuberculatus (deep depression) and C. phuketensis (shallow depression). The PSUZC-RT 2010.58 and THNHM 15378 specimens are two males of $C$. phuketensis, in which the precloacal grooves are shallow (all others are deep) and could result from their poor state of preservation; thus, the character of this specimen was not included in the present diagnostic characters of $C$. macrotuberculatus.

Evidence from both overlapping ranges of morphology and relatively low sequence divergence indicate that $C$. phuketensis is an inconsistent pattern variation of $C$. macrotuberculatus. We concluded that $C$. phuketensis should be treated as a junior synonym of $C$. macrotuberculatus based on the priority of names designated by International Code of Zoological Nomenclature (ICZN). Additional surveys should be conducted to determine their geographic distribution and the degree of variation and patterns of gene flow within this species.

\section{Acknowledgments}

This work was supported by grants from the Thailand Research Fund (DBG6080010) and the Center of Excellence on Biodiversity (BDC), Office of Higher Education Commission (BDC-PG4-160022). AA was supported by the Department of Zoology and International SciKU Branding (ISB), Faculty of Science, Kasetsart University and KT was supported by a grant from the Faculty of Science, Kasetsart University $\left(50^{\text {th }}\right.$ Anniversary of Faculty of Science). This research was approved by the Institutional Animal Care and Use Committee of Faculty of Science, Kasetsart University (project number ACKU61-SCI-006). The Department of National Parks, Wildlife and Plant Conservation, Thailand, granted permission to conduct the research. We thank Piyawat Sukon (superintendent of Khao Phra Thaeo Non-hunting Area), Kanchanapan Kamhang (superintendent of Tarutao National Park), and Chai Suvannachat (superintendent of Ton Nga Chang Wildlife Sanctuary) for facilitating the fieldwork. Wachara Sanguansombat and Sunchai Makchai (Thailand Natural History Museum) made specimens in their care available for study. Siriporn Yodthong, Natee Ampai, Akrachai Aksornneam and Piyawan Puanprapai assisted with fieldwork. This paper is contribution number 919 of the Auburn University Museum of Natural History.

\section{References}

Ampai N, Rujirawan A, Wood Jr PL, Stuart BL, Aowphol A (2019) Morphological and molecular analyses reveal two new insular species of Cnemaspis Strauch, 1887 (Squamata, 
Gekkonidae) from Satun Province, southern Thailand. ZooKeys 858: 127-161. https:// doi.org/10.3897/zookeys.858.34297

Grismer LL (2011) Lizards of Peninsular Malaysia, Singapore and Their Adjacent Archipelagos. Edition Chimaira, Frankfurt am Main-Germany, 728 pp.

Grismer LL, Ahmad N (2008) A new insular species of Cyrtodactylus (Squamata: Gekkonidae) from the Langkawi Archipelago, Kedah, Peninsular Malaysia. Zootaxa 1924: 53-68. https://doi.org/10.11646/zootaxa.1924.1.3

Grismer LL, Wood Jr PL, Anuar S, Grismer MS, Quah ES, Murdoch ML, Muin MA, Davis HR, Aguilar C, Klabacka R, Cobos AJ, Aowphol A, Site Jr JW (2016) Two new Benttoed Geckos of the Cyrtodactylus pulchellus complex from Peninsular Malaysia and multiple instances of convergent adaptation to limestone forest ecosystems. Zootaxa 4105(5): 401-429. https://doi.org/10.11646/zootaxa.4105.5.1

Grismer LL, Wood Jr PL, Anuar S, Quah ES, Muin MA, Mohamed M, Chan KO, Sumarli AX, Loredo AI, Heinz HM (2014) The phylogenetic relationships of three new species of the Cyrtodactylus pulchellus complex (Squamata: Gekkonidae) from poorly explored regions in northeastern Peninsular Malaysia. Zootaxa 3786(3): 359-381. https://doi.org/10.11646/ zootaxa.3786.3.6

Grismer LL, Wood Jr PL, Le MD, Quah ES, Grismer JL (2020) Evolution of habitat preference in 243 species of Bent-toed geckos (Genus Cyrtodactylus Gray, 1827) with a discussion of karst habitat conservation. Ecology and Evolution 10(24): 13717-13730. https://doi. org/10.1002/ece3.6961

Grismer LL, Wood Jr PL, Ngo VT, Murdoch ML (2015) The systematics and independent evolution of cave ecomorphology in distantly related clades of Bent-toed Geckos (Genus Cyrtodactylus Gray, 1827) from the Mekong Delta and islands in the Gulf of Thailand. Zootaxa 3980(1): 106-126. https://doi.org/10.11646/zootaxa.3980.1.6

Grismer LL, Wood Jr PL, Poyarkov NA, Le MD, Kraus F, Agarwal I, Oliver PM, Nguyen SN, Nguyen TQ, Karunarathna S, Welton LJ, Stuart BL, Luu VQ, Bauer AM, O’Connell KA, Quah ESH, Chan KO, Ziegler T, Ngo H, Nazarov RA, Aowphol A, Chomdej S, Suwannapoom C, Siler CD, Anuar S, Tri NV, Grismer JL (2021a) Phylogenetic partitioning of the third-largest vertebrate genus in the world, Cyrtodactylus Gray, 1827 (Reptilia; Squamata; Gekkonidae) and its relevance to taxonomy and conservation. Vertebrate Zoology 71: 101-154. https://doi.org/10.3897/vertebrate-zoology.71.e59307

Grismer LL, Wood Jr PL, Poyarkov NA, Le MD, Karunarathna S, Chomdej S, Suwannapoom C, Qi S, Liu S, Che J, Quah ES, Kraus F, Oliver PM, Riyanto A, Pauwels OSG, Grismer JL (2021b) Karstic Landscapes Are Foci of Species Diversity in the World's Third-Largest Vertebrate Genus Cyrtodactylus Gray, 1827 (Reptilia: Squamata; Gekkonidae). Diversity 13(5): e183. https://doi.org/10.3390/d13050183

Grismer LL, Wood Jr PL, Quah ES, Anuar S, Muin MA, Sumontha M, Ahmad N, Bauer AM, Wangkulangkul S, Grismer JL, Pauwels OS (2012) A phylogeny and taxonomy of the Thai-Malay Peninsula Bent-toed Geckos of the Cyrtodactylus pulchellus complex (Squamata: Gekkonidae): combined morphological and molecular analyses with descriptions of seven new species. Zootaxa 3520: 1-55. https://doi.org/10.11646/zootaxa.3520.1.1 
Grismer LL, Wood Jr PL, Thura MK, Quah ES, Murdoch ML, Grismer MS, Herr MW, Lin A, Kyaw H (2018) Three more new species of Cyrtodactylus (Squamata: Gekkonidae) from the Salween Basin of eastern Myanmar underscore the urgent need for the conservation of karst habitats. Journal of Natural History 52(19-20): 1243-1294. https://doi.org/10.108 0/00222933.2018.1449911

Huelsenbeck JP, Ronquist F (2001) MRBAYES: Bayesian inference of phylogenetic trees. Bioinformatics 17(8): 754-755. https://doi.org/10.1093/bioinformatics/17.8.754

Hurston H, Voith L, Bonanno J, Foufopoulos J, Pafilis P, Valakos E, Anthony N (2009) Effects of fragmentation on genetic diversity in island populations of the Aegean wall lizard Podarcis erhardii (Lacertidae, Reptilia). Molecular Phylogenetics and Evolution 52(2): 395-405. https://doi.org/10.1016/j.ympev.2009.03.028

Jang Y, Hahm EH, Lee H-J, Park S, Won Y-J, Choe CJ (2011) Geographic Variation in Advertisement Calls in a Tree Frog Species: Gene Flow and Selection Hypotheses. PLoS ONE 6(8): e23297. https://doi.org/10.1371/journal.pone.0023297

Kumar S, Stecher G, Li M, Knyaz C, Tamura K (2018) MEGA X: Molecular Evolutionary Genetics Analysis across computing platforms. Molecular Biology and Evolution 35(6): 1547-1549. https://doi.org/10.1093/molbev/msy096

Lanfear R, Frandsen PB, Wright AM, Senfeld T, Calcott B (2016) PartitionFinder 2: new methods for selecting partitioned models of evolution for molecular and morphological phylogenetic analyses. Molecular biology and evolution 34(3): 772-773. https://doi. org/10.1093/molbev/msw260

Lê S, Josse J, Husson F (2008) FactoMineR: An R Package for Multivariate Analysis. Journal of Statistical Software 25(1): 1-18. https://doi.org/10.18637/jss.v025.i01

Lleonart J, Salat J, Torres GJ (2000) Removing allometric effects of body size in morphological analysis. Journal of Theoretical Biology 205: 85-93. https://doi.org/10.1006/ jtbi.2000.2043

Macey JR, Larson A, Ananjeva NB, Papenfuss TJ (1997) Evolutionary shifts in three major structural features of the mitochondrial genome among iguanian lizards. Journal of Molecular Evolution 44(6):660-674. https://doi.org/10.1007/PL00006190

Mecke S, Kieckbusch M, Hartmann L, Kaiser H (2016) Historical considerations and comments on the type series of Cyrtodactylus marmoratus Gray, 1831, with an updated comparative table for the bent-toed geckos of the Sunda Islands and Sulawesi. Zootaxa 4175(4): 353-365. https://doi.org/10.11646/zootaxa.4175.4.5

Miller MA, Pfeiffer W, Schwartz T (2010) Creating the CIPRES Science Gateway for Inference of Large Phylogenetic Trees in Proceedings of the Gateway Computing Environments Workshop (GCE), 14 Nov. 2010, New Orleans, 8 pp. [assessed 6 September 2020] https://doi.org/10.1109/GCE.2010.5676129

Minh BQ, Nguyen MAT, von Haeseler A (2013) Ultrafast Approximation for Phylogenetic Bootstrap. Molecular Biology and Evolution 30(5): 1188-1195. https://doi.org/10.1093/ molbev/mst024

Murdoch ML, Grismer LL, Wood Jr PL, Neang T, Poyarkov NA, Ngo VT, Nazarov RA, Aowphol A, Pauwels OSG, Nguyen HN, Grismer JL (2019) Six new species of the 
Cyrtodactylus intermedius complex (Squamata: Gekkonidae) from the Cardamom Mountains and associated highlands of Southeast Asia. Zootaxa 4554(1): 1-62. https://doi. org/10.11646/zootaxa.4554.1.1

Nielsen SV, Oliver PM (2017) Morphological and genetic evidence for a new karst specialist lizard from New Guinea (Cyrtodactylus: Gekkonidae). Royal Society Open Science 4(11): e170781. https://doi.org/10.1098/rsos.170781

Pauwels OS, Nazarov RA, Bobrov VV, Poyarkov NA (2018) Taxonomic status of two populations of Bent-toed Geckos of the Cyrtodactylus irregularis complex (Squamata: Gekkonidae) with description of a new species from Nui Chua National Park, southern Vietnam. Zootaxa 4403(2): 307-335. https://doi.org/10.11646/zootaxa.4403.2.5

Quah ES, Grismer LL, Wood Jr PL, Sah SAM (2019) The discovery and description of a new species of Bent-toed Gecko of the Cyrtodactylus pulchellus complex (Squamata: Gekkonidae) from the Langkawi Archipelago, Kedah, Peninsular Malaysia. Zootaxa 4668(1): 51-75. https://doi.org/10.11646/zootaxa.4668.1.3

RStudio Team (2018) RStudio: Integrated Development for R. RStudio, Inc., Boston. http:// www.rstudio.com/

Rambaut A, Drummond AJ, Xie D, Baele G, Suchard MA (2018) Posterior summarization in Bayesian phylogenetics using Tracer 1.7. Systematic Biology 67(5): 901-904. https://doi. org/10.1093/sysbio/syy032

Riyanto A, Mulyadi M, Mcguire JA, Kusrini MD, Febylasmia F, Basyir IH, Kaiser H (2017) A new small bent-toed gecko of the genus Cyrtodactylus (Squamata: Gekkonidae) from the lower slopes of Mount Tambora, Sumbawa Island, Indonesia. Zootaxa 4242(3): 517-528. https://doi.org/10.11646/zootaxa.4242.3.5

Ronquist F, Teslenko M, Van Der Mark P, Ayres DL, Darling A, Höhna S, Larget B, Liu L, Suchard MA, Huelsenbeck JP (2012) MrBayes 3.2: efficient Bayesian phylogenetic inference and model choice across a large model space. Systematic Biology 61: 539-542. https://doi.org/10.1093/sysbio/sys029

Sumontha M, Pauwels OS, Kunya K, Nitikul A, Samphanthamit P, Grismer LL (2012) A new forest-dwelling gecko from Phuket Island, Southern Thailand, related to Cyrtodactylus macrotuberculatus (Squamata: Gekkonidae). Zootaxa 3522: 61-72. https://doi.org/10.11646/ zootaxa.3522.1.4

Thorpe RS (1975) Quantitative handling of characters useful in snake systematics with particular reference to interspecific variation in the Ringed Snake Natrix natrix (L.). Biological Journal of the Linnean Society 7: 27-43. https://doi.org/10.1111/j.1095-8312.1975. tb00732.x

Thorpe RS (1983) A review of the numerical methods for recognized and analysing racial differentiation. In: Felsenstein J (Ed.) Numerical Taxonomy. Berlin Heidelberg: Springer, 404-423. https://doi.org/10.1007/978-3-642-69024-2_43

Trifinopoulos J, Nguyen LT, von Haeseler A, Minh BQ (2016) W-IQ-TREE: a fast online phylogenetic tool for maximum likelihood analysis. Nucleic Acids Research 44 (W1): W232W235. https://doi.org/10.1093/nar/gkw256

Turan C (1999) A note on the examination of morphometric differentiation among fish populations: the truss system. Turkish Journal of Zoology 23: 259-263. 
Uetz P, Freed P, Hošek J (2020) The Reptile Database: http://www.reptile-database.org [accessed 8 February 2021]

Wilcox TP, Zwickl DJ, Heath TA, Hillis DM (2002) Phylogenetic relationships of the dwarf boas and a comparison of Bayesian and bootstrap measures of phylogenetic support. Molecular Phylogenetics and Evolution 25: 361-371. https://doi.org/10.1016/S10557903(02)00244-0

Wood Jr PL, Grismer LL, Muin MA, Anuar S, Oaks JR, Sites Jr JW (2020) A new potentially endangered limestone-associated Bent-toed Gecko of the Cyrtodactylus pulchellus (Squamata: Gekkonidae) complex from northern Peninsular Malaysia. Zootaxa 4751(3): 437-460.

Wood Jr PL, Heinicke MP, Jackman TR, Bauer AM (2012) Phylogeny of bent-toed geckos (Cyrtodactylus) reveals a west to east pattern of diversification. Molecular phylogenetics and evolution 65(3): 992-1003. https://doi.org/10.1016/j.ympev.2012.08.025 